\begin{tabular}{|c|l|}
\hline Title & Free surface flows under impacting droplets \\
\hline Author(s) & Watanabe, Y asunori; Saruwatari, Ayunii; Ingram, David M. \\
\hline Citation & $\begin{array}{l}\text { Journal of Computational Physics, 227(4), 2344.2365 } \\
\text { https://doi.org/10.1016/.jcp.2007.10.020 }\end{array}$ \\
\hline Issue Date & 2008-02-01 \\
\hline Doc URL & http://hdl.handle.net/2115/33768 \\
\hline Type & article (author version) \\
\hline File Information & yjcph18714huscap.pdf \\
\hline
\end{tabular}

Instructions for use 


\title{
Free-surface Flows Under Impacting Droplets
}

\author{
Yasunori Watanabe, ${ }^{a}$ Ayumi Saruwatari, ${ }^{a}$ David M. Ingram ${ }^{b}$ \\ a School of Engineering, Hokkaido University, North 13 West 8, Sapporo 060-8628, \\ Japan \\ b Institute for Energy Systems, The University of Edinburgh, Kings Building, \\ Mayfield Road, Edinburgh EH9 3JL, UK
}

\begin{abstract}
A numerical method which fulfils the free-surface boundary conditions and extrapolates the fluid velocity in to empty grid cells outside the fluid region on a fixed Cartesian grid system is presented. The complex, three-dimensional, vortex structures formed via surface/vortex interaction and induction between vortices have been computed using the proposed technique implemented within a level-set method for both vertical and oblique droplet impacts in incompressible fluids. The present results have been validated through numerical tests which confirm zero tangential shear at the free-surface and comparisons with experimental observations of cavity and vortex ring formation underneath the impact location. In some cases, transitions from a concentric vortex ring to a fully three-dimensional vortex structure has been confirmed. Whilst the primary vortex ring is initiated at the highly curved contact surface between the droplet and receiving surface, azimuthal instabilities are manifested in the shear layer around the cavity crater developing after the vertical impact, resulting in axial counter-rotating vorticity between the cavity and descending vortex ring. Underlying mechanisms which induce local deformation of the free-surface, creating a so-called scar, due to the sub-surface vortices at the oblique impacts are also discussed.
\end{abstract}

Key words: droplet impact, vortex ring, free-surface boundary condition, level-set method

\section{Introduction}

A single droplet splashing onto a still water surface produces vorticity on the highly curved contact surface between the droplet and receiving free-surface, normally leading to the formation of a ring vortex separated from the surface boundary layer. Finger jets are sometimes observed for forming a crown along 
the circumference of the radial cavity crater, created when the drop has penetrated into the surface(Yarin , 2006). As the cavity begins to collapse a vertical jet is formed at the centre of the cavity due to the radial pressure gradients. The vertical jet projects upwards before fragmenting into droplets. The initiation and evolution of the vortex structure under droplet impacts arise through surface-vortex interactions (Peck and Sigurdson , 1994). The initial vorticity of the primary vortex ring is produced at the highly curved droplet/surface contact where tangential shear vanishes (Longuet-Higgins , 1992). The primary vortex ring develops around the cavity wall and is subsequently ejected from the bottom surface of the rising cavity. It has been observed that counterrotating vortex pairs are produced around the primary vortex ring and are vertically stretched between the centre of the ring and cavity bottom, resulting in a complex, axisymmetric, three-dimensional, vortex structure involving the vortex rings wrapping the vortex loops (Peck and Sigurdson, 1994). A recent experimental study by Leneweit et al. (2005) characterised the formation of capillary waves ahead of the progressing droplet front, the distortion of the cavity and droplet immersion under varying impact angles and Weber number, $W e$. The formation of anchor-like patterns of droplet immersion observed by Leneweit et al. (2005) suggest the presence of complex vortex structures involving the droplet fluid beneath the free-surface.

Many attempts to compute both free-surface and interface flows have been undertaken (see a review by Scardovelli and Zalenski , 1999). The Volume of fluid (VOF) method has been commonly used for the free-surface computations in a fixed Cartesian grid system (Hirt and Nichols , 1981), however, a high-order scheme needs to be introduced for reconstructing highly curved surface. Gueyffer et al. (Gueyffier et al. , 1999) proposed an interface tracking technique based on a combination of VOF and PLIC (piece-wise linear interface calculation) for computing droplet sedimentation and rising bubbles and the jetting process during the drop impacts on a thin liquid film (Jeoung and Moffat , 1992). A front tracking method (Esmaeeli and Tryggvason , 1998, 1999) has also been used to capture and reconstruct a sharp interface in bubble flows and splashing droplets (Sousa et al. , 2004). The level-set method (Osher and Sethian, 1988) is one of the most powerful technique available to determine implicit surfaces within a fixed grid system and has many applications for two-phase flows with complicated shapes of interfaces (e.g. Susman et al. , 1994; Chang et al. , 1996). A recent review by Losasso et al. (2005) introduced many versions of the level-set technique combined with another methods such as VOF and AMR (Adaptive Mesh Refinement) for improving mass conservation and maintaining a sharp interfaces. The ghost fluid method also provides a robust technique to suppress numerical oscillations in pressure and velocity due to two-phase contact discontinuities (Fedkiw et al. , 1999). In the case of droplet impacts, the reciprocal effects of gravity, surface-tension and viscous stress on and near the free-surface must all be considered since they substantially determine the nature of the free-surface dynamics. Further- 
more the dynamic free-surface conditions, zero tangential shear (eqs. 8 and 9) and normal jump conditions (eq. 7), must be appropriately satisfied in order to reproduce the evolution of vortices, intensified within the surface boundary layer throughout the impacting process. In most level-set and VOF computations for inter-facial flows, fluid density is numerically smoothed across the surface in order to avoid numerical instabilities, and the surface tension is incorporated in to the momentum equation as a source term using a smearout numerical Delta function over the grid cells which span the surface (e.g. Susman et al. , 1994; Morton et al. , 2000). However, such surface models are unable to model physical features of the sub-surface flows governed by surface dynamics under the droplet impacts unless the very thin mass boundary layer is sufficiently resolved, since the dynamic jump conditions at the surface are cannot be satisfied on the smeared-out surface.

Both the splashing jets of breaking waves and resulting turbulent flows have been computed using large eddy simulation (Watanabe and Saeki , 1999; Christensen and Deigaard , 2001; Lubin et al. , 2006). Using the same computational methods a mechanism to form three-dimensional vortices via instabilities on a shear layer formed in a two-dimensional vortex array within the surf zone has recently been identified by Watanabe et al. (2005). However, the detailed surface-vortex interactions are not guaranteed to be appropriately modelled in these computations since simplified free-surface boundary conditions were employed. Popinet and Zaleski (2002) presented an extrapolation technique for the liquid-phase velocity to empty grid cells inside an air bubble, which implicitly fulfils the zero tangential shear condition at the free-surface. In this method, the velocity outside the surface is approximated by a linear function with near-surface velocity to minimize a penalty term for approximately ensuring zero tangential condition on the surface. This is a reasonable approach for computing the advection of the free surface with a local velocity field which approximately satisfies the dynamic boundary condition and the evolution of collapsing bubble shapes has been computed using this method.

In the study of droplet impacts, however, it is very important to reproduce surface-vortex interactions in the computations, since the initial formation of a vortex ring at the droplet contact and its subsequent evolution within the surface shear layer beneath the cavity are strongly influenced by local surface deformations including capillary wave propagating on the cavity walls (Morton et al. , 2000). Furthermore, when counter-rotating vortices are located beneath the water surface horizontally, the free-surface is deformed locally - the convergence of the flow on the surface over the cylinders results in a formation of the surface cusp, while inverse counter-rotating cylinders induce a diverging flow at the surface which is lifted by the upwelling flow between (Jeoung and Moffat , 1992). Accurate computation of flow fields involving surface-vortex interactions is achieved by accurately estimating surface velocity gradients from the surface shear flow, thus fulfilling the dynamic boundary 
condition in three-dimensional space.

In this study, an alternative technique to fulfil the free-surface dynamic boundary condition via quadratic polynomial extrapolation into the empty grid cells on a fixed three-dimensional grid system is proposed. This approach has the advantage that it is able to reflect local features of velocity gradients within the curved surface shear layers.

The technique has been applied to local free-surface deformation and evolution of the sub-surface vortices in a series of droplet impacts. Both the computational and experimental surface deformations during the impacting process are compared, and underlying mechanisms for organising three-dimensional vortex structures in both vertical and oblique droplet impacts are discussed on the basis of strain and vorticity dynamics.

This paper is organised as follows. The computational method is described in $\S 2$. The computations are validated through numerical tests which confirm zero tangential shear on the surface in $\S 3$. In $\S 4$, sequential free-surface deformations and vortex organisations under both vertical and oblique droplet impacts are compared with visual observations, and major features of the impacting process in terms of surface-vortex interactions are discussed. The results are summarised in $\S 5$.

\section{$2 \quad$ Numerical method}

The droplet impacts considered in this study will induce turbulence since the droplet Reynolds number is in the range 2000 to 5400. Consequently large eddy simulation is employed to describe the turbulent flows. Large eddy simulation based on a two-fluid model has been performed for studying turbulent energy transfer across a gas-liquid interface by Labourasse et al. (2007) and turbulent interfacial shear flows by Reboux et al. (2006). In this study, the free-surface turbulent flows of a splashing droplet have been modelled using an identical underlying model and computational scheme to that described by Watanabe et al. (2005), a model which has been used in the previous study of turbulence under breaking waves and splashing jets, with the exception of the proposed method for computations related to free-surfaces.

The Navier-Stokes equations under the incompressible assumption, $\nabla \cdot \boldsymbol{u}^{*}=0$, are;

$$
\frac{D^{*} \boldsymbol{u}^{*}}{D t^{*}}=-\frac{1}{\rho^{*}} \nabla^{*} p^{*}+\nabla^{*} \cdot \tau_{0}^{*}+\boldsymbol{g}^{*}
$$


where $\boldsymbol{u}^{*}$ denotes the fluid velocity, $p^{*}$ is the pressure, $\boldsymbol{\tau}_{\mathbf{0}}^{*}$ is the viscous stress tensor $\left(=2 \nu_{0}^{*} \boldsymbol{S}^{*}\right.$, where $\nu_{0}^{*}$ is the kinematic viscosity and $\boldsymbol{S}^{*}$ is the strain tensor), and $\boldsymbol{g}^{*}$ is the gravitational acceleration. All variables in equation (1) are normalised with reference to the droplet diameter $\left(d^{*}\right)$, the initial droplet impact velocity $\left(U^{*}\right)$, and the fluid density $\left(\rho^{*}\right): \boldsymbol{u}=\boldsymbol{u}^{*} / U^{*}, p=p^{*} / \rho^{*} U^{*}, \boldsymbol{g}=$ $\boldsymbol{g}^{\prime} / F r^{2}, \boldsymbol{\tau}_{\mathbf{0}}=2 \boldsymbol{S}^{*} d^{*} / U^{*} R e, t=U^{*} t^{*} / d^{*}$ and $\boldsymbol{x}=\boldsymbol{x}^{*} / d^{*}$, where $\boldsymbol{g}^{\prime}$ is the unit gravity vector, $\operatorname{Fr}\left(=U^{*} / \sqrt{g^{*} d^{*}}\right)$ is the Froude number and $\operatorname{Re}\left(=\nu_{0}^{*} / U^{*} d^{*}\right)$ is the Reynolds number. The dimensionless form of equation (1) is written by

$$
\frac{D \boldsymbol{u}}{D t}=-\nabla p+\nabla \cdot \boldsymbol{\tau}_{\mathbf{0}}+\frac{1}{F r^{2}} \boldsymbol{g}^{\prime}
$$

The differential operators in equation (2) are the material derivative $\frac{D}{D t}=$ $\left.\frac{\partial}{\partial t}+u \frac{\partial}{\partial x}+v \frac{\partial}{\partial y}+w \frac{\partial}{\partial z}\right)$.

For any arbitrary variable $\psi$, the grid-scale (GS) variable $\bar{\psi}$ is obtained by the filtering operation

$$
\bar{\psi}=\int G\left(\boldsymbol{x}-\boldsymbol{x}^{\prime}\right) \psi\left(\boldsymbol{x}^{\prime}\right) d^{3} \boldsymbol{x}^{\prime}
$$

where $G$ is the filter kernel, and the subgrid-scale (SGS) component is then defined as $\psi^{\prime}=\psi-\bar{\psi}$.

After performing the filtering operations, equation (2) becomes

$$
\frac{D_{f} \overline{\boldsymbol{u}}}{D t}=-\nabla \bar{p}-\nabla \cdot \boldsymbol{\tau}+\nabla \cdot \overline{\boldsymbol{\tau}_{\mathbf{0}}}+\frac{1}{F r^{2}} \boldsymbol{g}^{\prime}
$$

where $\boldsymbol{\tau}$ denotes the SGS stress tensor, $\frac{D_{f}}{D t}=\frac{\partial}{\partial t}+\bar{u} \frac{\partial}{\partial x}+\bar{v} \frac{\partial}{\partial y}+\bar{w} \frac{\partial}{\partial z}$ is the filtered material derivative. The second and third terms on the right hand side of equation (4) are described by the SGS viscosity form

$$
\boldsymbol{\tau}-\frac{1}{3}(\boldsymbol{\tau}: \boldsymbol{I}) \boldsymbol{I}-\overline{\boldsymbol{\tau}_{0}}=-2 \nu \overline{\boldsymbol{S}}
$$

in tensor form, where $\boldsymbol{I}$ is the unit dyadic. The SGS viscosity model based on renormalisation group theory (Yakhot and Orszag , 1986) is

$$
\nu=\frac{1}{R e}\left[1+H\left(c_{s}^{2} \Delta^{4} \nu R e^{3}(2 \overline{\boldsymbol{S}}: \overline{\boldsymbol{S}})-C\right)\right]^{1 / 3}
$$

where $H$ is the Heaviside unit step function $(H(x)=0$ if $x \leq 0, H(x)=x$ if $x>0), c_{s}$ is the Smagorinsky constant, the constant $C=75$, and $\Delta$ is the filter 
scale. The dimensionless viscosity coefficient $\nu$ is computed from equation (6) at each time step, using Newton-Raphson iteration. This SGS viscosity model for transient turbulent flows with splashing jets of breaking waves has been validated in Watanabe et al. (2005).

The jump condition for momentum conservation across a free-surface leads the normal and tangential dynamic boundary conditions:

$$
\begin{gathered}
\bar{p}_{s f}+2 \nu \frac{\partial \overline{u_{n}}}{\partial n}=\frac{\kappa}{W e}, \\
\nu\left(\frac{\partial \overline{u_{n}}}{\partial r}+\frac{\partial \overline{u_{r}}}{\partial n}\right)=0, \\
\nu\left(\frac{\partial \overline{u_{n}}}{\partial s}+\frac{\partial \overline{u_{s}}}{\partial n}\right)=0,
\end{gathered}
$$

where $\bar{p}_{s f}$ is the surface pressure, $\kappa$ is the dimensionless surface curvature, $W e\left(=\rho^{*} U^{* 2} d^{*} / \sigma^{*}\right)$ is the Weber number, $\sigma^{*}$ is the coefficient of surface tension, and $\overline{u_{n}}, \overline{u_{r}}$ and $\overline{u_{s}}$ are resolved normal and two tangential velocities, respectively. The normal jump condition (7) is directly used at surfaces in iterative computations of a Poisson equation for pressure (17), and the two tangential shear conditions $(8,9)$ are fulfilled as explained in $§ 2.3$.

\subsection{Level-set approach}

The location of the free-surface is defined in terms of an advected scalar, $\phi$, which is defined as a signed distance function from the interface. The, socalled, level-set equation, is simply a hyperbolic conservation law for $\phi$;

$$
\frac{D_{f} \phi}{D t}=0
$$

It should be noted that $\phi$ is taken to be positive inside the fluid and negative outside the fluid. The specific location of the free surface is defined by the set of points $\{(\boldsymbol{x}, t): \phi(\boldsymbol{x}, t)=0\}$. The advection equation (10) is updated by the Cubic Interpolation Polynomials (CIP) method, which is a quasi-Lagrangian technique for computing advection based on a third-order polynomial interpolation within a cell (see details in Watanabe and Saeki , 1999, 2002; Watanabe et al. , 2005). 
Since $\nabla \phi$ is perpendicular to the iso-contour $\phi=0$, the unit outward normal vector $\boldsymbol{n}$ and the curvature $\kappa$ of the surface are given by

$$
\boldsymbol{n}=-\frac{\nabla \phi}{|\nabla \phi|}
$$

and

$$
\kappa=\nabla \cdot \boldsymbol{n}
$$

respectively. The two unit tangential vectors $\boldsymbol{t}$ and $\boldsymbol{s}$ on the surface can now be estimated via

$$
\begin{aligned}
& \boldsymbol{t}=\frac{\boldsymbol{u}_{\text {nearest }}-\left(\boldsymbol{n} \cdot \boldsymbol{u}_{\text {nearest }}\right) \boldsymbol{n}}{\boldsymbol{n} \cdot \boldsymbol{u}_{\text {nearest }}}, \\
& \boldsymbol{s}=\boldsymbol{t} \times \boldsymbol{n}
\end{aligned}
$$

where $\boldsymbol{u}_{\text {nearest }}$ is the fluid velocity at the nearest grid cell to the surface. The surface tension and velocity derivatives in the free-surface boundary conditions (7) - (9) can be represented by the level-set function using relations of (11) (14).

To ensure numerical stability it is usual, in computations based on the levelset approach, to smooth the fluid density and viscosity over a finite interface thickness $\delta$, normally containing between 3 and 5 grid cells, and to model the surface tension as a source term varying over multiple grid cells across the free-surface (e.g. Susman et al. , 1994). Although this smeared-out surface model may be advantageous for avoiding numerical instabilities, it makes it impossible to accurately compute the dynamics of the free-surface unless very fined grids are used (with cell spacing of the order of $\mu \mathrm{m}$ ) so as to resolve a thin mass boundary layer. In order to avoid the excessive cost associated with such fine grid resolution, a reasonable approximation of the free-surface boundary conditions needs to be determined so the significant surface-vortex interactions which occur during the droplet impacting process can be simulated.

One further difficulty is that $\phi$ needs to be reinitialised in order to maintain $\phi$ property of being a signed distance function. In the present computations the reinitialisation method proposed by Susman and Fatemi (1999), which guarantees no movement of the surface location at $\phi=0$, has been adopted. 


\subsection{Numerical method for free-surface flow}

The present computations are based on a finite difference discretisation scheme on a fixed, staggered grid system. A fractional two-step, predictor-corrector, method is applied to the discretized forms of equation (4):

$$
\begin{aligned}
& \frac{\overline{\boldsymbol{u}}^{n+1 / 2}-\overline{\boldsymbol{u}}^{n}}{\Delta t}=-\nabla \bar{p}^{n+1 / 2}+\boldsymbol{R}^{n+1 / 2}, \\
& \frac{\overline{\boldsymbol{u}}^{n+1}-\overline{\boldsymbol{u}}^{n+1 / 2}}{\Delta t}+\overline{\boldsymbol{u}}^{n+1 / 2} \cdot\left(\boldsymbol{\nabla} \overline{\boldsymbol{u}}^{n+1 / 2}\right)=0,
\end{aligned}
$$

where the right hand side of equation (4) $\boldsymbol{R}=-\boldsymbol{\nabla} \cdot \boldsymbol{\tau}+\boldsymbol{\nabla} \cdot \overline{\boldsymbol{\tau}_{\mathbf{0}}}+\boldsymbol{g}^{\prime} / F r^{2}$. In the predictor step, the pressure $\bar{p}^{n+1 / 2}$ is obtained through iterative computation of the Poisson pressure equation (17) with the normal dynamic surface boundary condition (7). The pressure gradient $\nabla \bar{p}^{n+1 / 2}$ in equation (15) is also determined with the normal condition (7) using equation (19).

The flow variables, $\boldsymbol{u}$, are estimated at the half time level using equation (15). The predicted values are updated via the nonlinear advection equation (16) using CIP method.

By taking divergence of equation (15) and setting $\boldsymbol{\nabla} \cdot \overline{\boldsymbol{u}}^{n+1 / 2}=0$, to ensure free divergence field for incompressible fluid, the Poisson equation for pressure can be obtained:

$$
\nabla^{2} \bar{p}^{n+1 / 2}=\frac{\boldsymbol{\nabla} \cdot \overline{\boldsymbol{u}}^{n}}{\Delta t}+\nabla \cdot \boldsymbol{R}^{n+1 / 2}
$$

The second derivative of pressure in equation (17) is discretized on a staggered grid $(i, j, k)$ adjacent to the free surface. The second order accurate discretizaton satisfies the normal surface boundary condition (7):

$$
\left.\frac{\partial^{2} \bar{p}}{\partial x^{2}}\right|_{i, j, k}=\frac{2\left(\Delta x^{-} \bar{p}^{+}+\Delta x^{+} \bar{p}^{-}-\left(\Delta x^{+}+\Delta x^{-}\right) \bar{p}_{i, j, k}\right)}{\Delta x^{+} \Delta x^{-}\left(\Delta x^{+}+\Delta x^{-}\right)}
$$

where

$$
\left\{\begin{array}{rlrl}
\Delta x^{ \pm} & =\Delta x \phi_{i, j, k} /\left(\phi_{i . j . k}-\phi_{i \pm 1, j, k}\right), \bar{p}^{ \pm} & =\bar{p}_{s f} & \left(\phi_{i, j, k}>0, \phi_{i \pm 1, j, k}<0\right) \\
\Delta x^{ \pm}=\Delta x, & \bar{p}^{ \pm}=\bar{p}_{i \pm 1, j, k} & \left(\phi_{i, j, k}>0, \phi_{i \pm 1, j, k} \geq 0\right)
\end{array}\right.
$$

$\Delta x$ is the grid spacing in the $x$-axis and the surface pressure $\bar{p}_{s f}$ is directly substituted from the normal dynamic boundary condition (7) (see figure 1, 

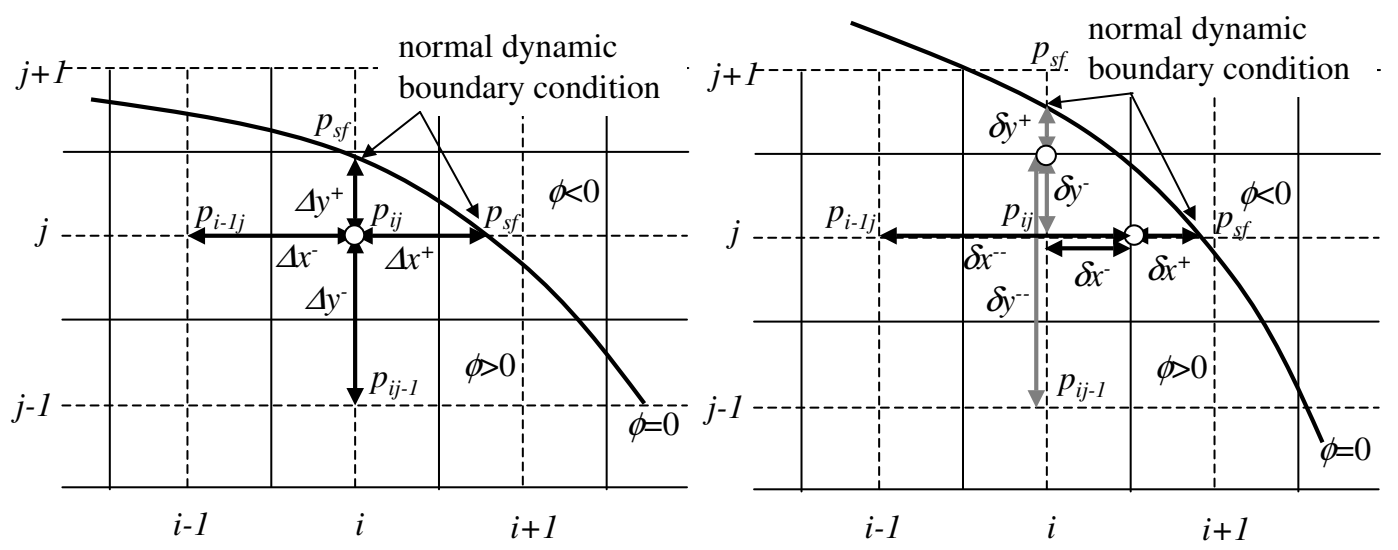

Fig. 1. Discritization of derivatives of the pressure near free-surfaces (left; second derivative, right; first derivative).

left). The other derivatives with respect to $y$ and $z$ are also discretized in the same way. Equation (17) is solved iteratively using a multi-grid method based on an irregular-star method (Chang and Street , 1970).

The pressure gradient in a grid cell adjacent to a surface needs to be defined when computing equation (15). It is defined at cell faces on a fixed staggered grid system (e.g. grid location at $(i+1 / 2, j, k)$ for horizontal velocity), and is also discretized using a second order accuracy approximation (see figure 1, right):

$$
\left.\frac{\partial \bar{p}}{\partial x}\right|_{i+1 / 2, j, k}=a \bar{p}_{i-1, j, k}+b \bar{p}_{i, j, k}+c \bar{p}_{s f}
$$

where $a=\frac{\delta x^{+}-\delta x^{-}}{\left(\delta x^{-}-\delta x^{--}\right)\left(\delta x^{--}+\delta x^{+}\right)}, b=\frac{\delta x^{--}-\delta x^{+}}{\left(\delta x^{-}-\delta x^{--}\right)\left(\delta x^{-}+\delta x^{+}\right)}, c=$ $\frac{\delta x^{--}-\delta x^{-}}{\left(\delta x^{--}+\delta x^{+}\right)\left(\delta x^{-}+\delta x^{+}\right)}$, and $\bar{p}_{s f}$ is the surface pressure obtained using the normal free-surface condition (7). $\delta x^{-}=\Delta x / 2$ and $\delta x^{--}=3 \Delta x / 2$. If $\phi_{i, j, k}>0$ and $\phi_{i \pm 1, j, k}<0, \delta x^{+}=\Delta x \phi_{i, j, k} /\left(\phi_{i}-\phi_{i \pm 1, j, k}\right)-\Delta x / 2$, otherwise $\delta x^{+}=$ $\Delta x / 2$ since there is no free-surface between the grid location $(i+1 / 2, j, k)$ and $(i+1, j, k))$. When there is no free-surface within the adjacent cell, the pressure gradient (19) reduces to a simple central difference approximation. The other derivatives are also evaluated in the same way.

\subsection{A Numerical procedure to satisfy the zero-tangential shear condition}

In the following sections, any filtered GS variable is simply described as a form without the over line. Assuming the velocity gradients on and near the 
free-surface have following relation:

$$
\frac{\partial u_{i}\left(\boldsymbol{x}_{s}+\boldsymbol{\xi}\right)}{\partial x_{j}}=\frac{\partial u_{i}^{s}\left(\boldsymbol{x}_{s}\right)}{\partial x_{j}}+f_{i j}(\boldsymbol{\xi})
$$

where $\boldsymbol{\xi}=(\xi, \eta, \zeta)$ is the position vector from the surface location $\boldsymbol{x}_{s}$ and $f_{i j}(\boldsymbol{\xi})$ is the correction function. A linear equation $f_{i j}(\boldsymbol{\xi})=a_{i j}+b_{i j} \xi+c_{i j} \eta+d_{i j} \zeta$ is chosen to approximate spatial variations of the velocity gradients, where $a_{i j}, b_{i j}, c_{i j}$ and $d_{i j}$ are local constants. Substituting equation (20) into the zeroshear tangential boundary conditions (8) and (9), we have

$$
\nu \sum_{i, j=1,2,3}\left(\left(\frac{\partial u_{i}\left(\boldsymbol{x}_{s}+\boldsymbol{\xi}\right)}{\partial x_{j}}-f_{i j}(\boldsymbol{\xi})\right)+\left(\frac{\partial u_{j}\left(\boldsymbol{x}_{s}+\boldsymbol{\xi}\right)}{\partial x_{i}}-f_{j i}(\boldsymbol{\xi})\right)\right) n_{j} t_{i}=0
$$

and

$$
\nu \sum_{i, j=1,2,3}\left(\left(\frac{\partial u_{i}\left(\boldsymbol{x}_{s}+\boldsymbol{\xi}\right)}{\partial x_{j}}-f_{i j}(\boldsymbol{\xi})\right)+\left(\frac{\partial u_{j}\left(\boldsymbol{x}_{s}+\boldsymbol{\xi}\right)}{\partial x_{i}}-f_{j i}(\boldsymbol{\xi})\right)\right) n_{j} s_{i}=0 .
$$

In a matrix expression, these can be written by

$$
\begin{aligned}
& \boldsymbol{T}_{t} \boldsymbol{A}=B_{t}, \\
& \boldsymbol{T}_{s} \boldsymbol{A}=B_{s},
\end{aligned}
$$

where $\boldsymbol{T}_{t}=\left[2 n_{1} t_{1}, 2 n_{1} t_{1} \xi, 2 n_{1} t_{1} \eta, 2 n_{1} t_{1} \zeta \ldots\left(n_{i} t_{j}+n_{j} t_{i}\right),\left(n_{i} t_{j}+n_{j} t_{i}\right) \xi\right.$, $\left.\left(n_{i} t_{j}+n_{j} t_{i}\right) \eta,\left(n_{i} t_{j}+n_{j} t_{i}\right) \zeta, \ldots 2 n_{3} t_{3}, 2 n_{3} t_{3} \xi, 2 n_{3} t_{3} \eta, 2 n_{3} t_{3} \zeta\right]$,

$\boldsymbol{T}_{s}=\left[2 n_{1} s_{1}, 2 n_{1} s_{1} \xi, 2 n_{1} s_{1} \eta, 2 n_{1} s_{1} \zeta \ldots\left(n_{i} s_{j}+n_{j} s_{i}\right),\left(n_{i} s_{j}+n_{j} s_{i}\right) \xi\right.$,

$\left.\left(n_{i} s_{j}+n_{j} s_{i}\right) \eta,\left(n_{i} s_{j}+n_{j} s_{i}\right) \zeta, \ldots 2 n_{3} s_{3}, 2 n_{3} s_{3} \xi, 2 n_{3} s_{3} \eta, 2 n_{3} s_{3} \zeta\right]$,

$\boldsymbol{A}=\left[a_{11}, b_{11}, c_{11}, d_{11} \ldots a_{i j}, b_{i j}, c_{i j}, d_{i j}, \ldots \ldots a_{33}, b_{33}, c_{33}, d_{33}\right]^{T}$,

$B_{t}=\sum_{i, j=1,2,3}\left(\frac{\partial u_{i}}{\partial x_{j}}+\frac{\partial u_{j}}{\partial x_{i}}\right) n_{j} t_{i}$

and

$B_{s}=\sum_{i, j=1,2,3}\left(\frac{\partial u_{i}}{\partial x_{j}}+\frac{\partial u_{j}}{\partial x_{i}}\right) n_{j} s_{i}$.

The coefficient matrix $\boldsymbol{A}$ can be approximated using the method of least squares, using $N$ velocity gradients on the nearest $N$ grid cells to the surface location $\left(\boldsymbol{x}=\boldsymbol{x}_{s}\right)$ in the fluid region:

$$
\boldsymbol{T A}=\boldsymbol{B},
$$


where

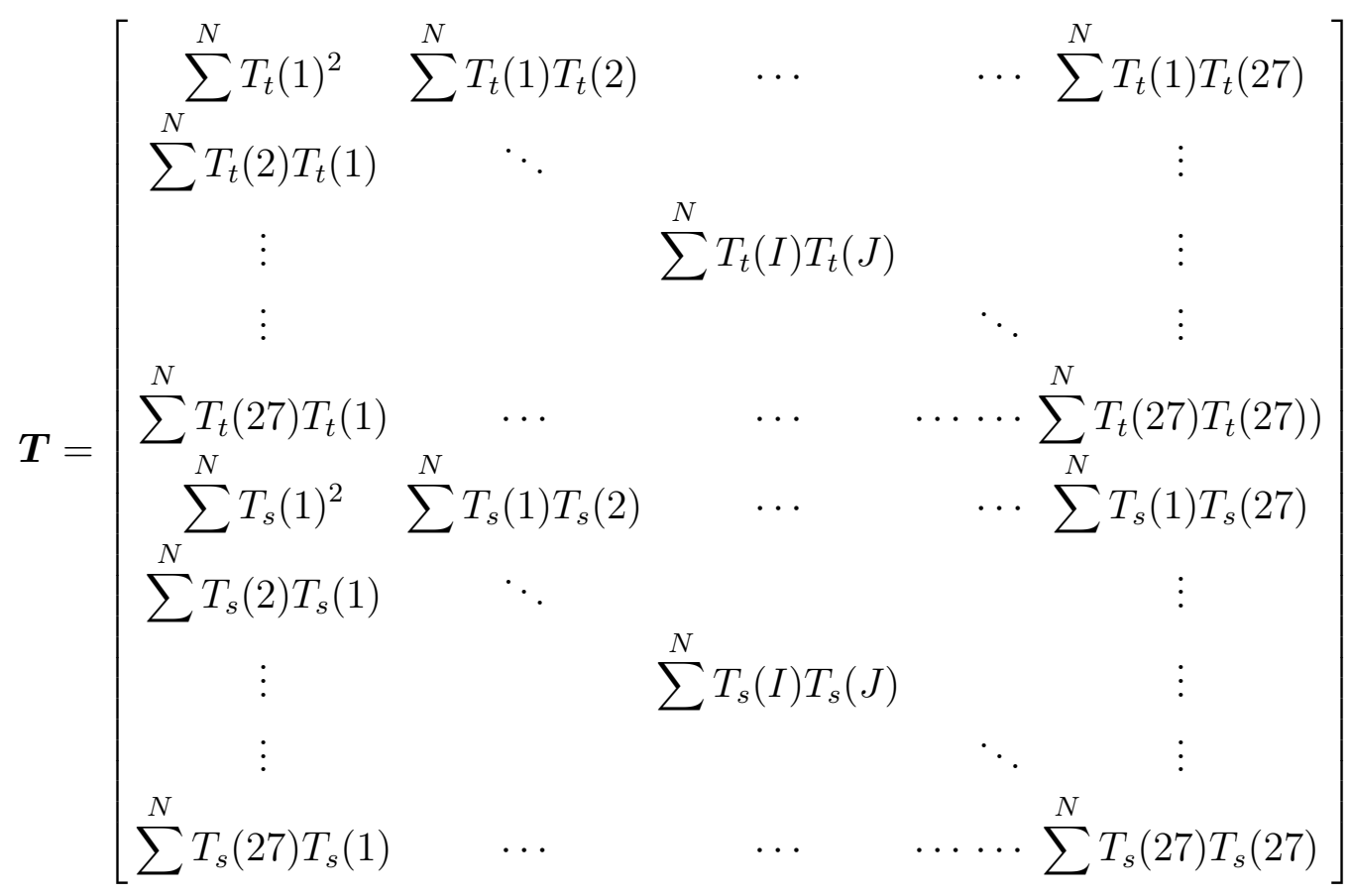

and

$$
\begin{aligned}
& \boldsymbol{B}=\left[\sum^{N} \sum_{i, j=1,2,3}\left(\frac{\partial u_{i}}{\partial x_{j}}+\frac{\partial u_{j}}{\partial x_{i}}\right) n_{j} t_{i} T_{t}(1) \cdots \sum_{i, j=1,2,3}^{N} \sum_{\partial x_{j}}\left(\frac{\partial u_{i}}{\partial x_{i}}\right) n_{j} t_{i} T_{t}(I)\right. \\
& \cdots \sum_{i, j=1,2,3}^{N}\left(\frac{\partial u_{i}}{\partial x_{j}}+\frac{\partial u_{j}}{\partial x_{i}}\right) n_{j} t_{i} T_{t}(27), \sum_{i, j=1,2,3}^{N}\left(\frac{\partial u_{i}}{\partial x_{j}}+\frac{\partial u_{j}}{\partial x_{i}}\right) n_{j} t_{i} T_{s}(1) \cdot(27) \\
& \left.\sum_{i, j=1,2,3}^{N}\left(\frac{\partial u_{i}}{\partial x_{j}}+\frac{\partial u_{j}}{\partial x_{i}}\right) n_{j} t_{i} T_{s}(I) \cdots \sum_{i, j=1,2,3}^{N}\left(\frac{\partial u_{i}}{\partial x_{j}}+\frac{\partial u_{j}}{\partial x_{i}}\right) n_{j} t_{i} T_{s}(27)\right]
\end{aligned}
$$

This, sparse, matrix can be computed using a singular value decomposition (SVD). It should be noted that, to ensure a sufficiently good approximation, the number of grid cells sampled, $N>6$.

The velocity near the surface at $\boldsymbol{x}=\boldsymbol{x}_{s}+\boldsymbol{\xi}$ is also approximated by Taylor series with respect to the surface location $\left(\boldsymbol{x}_{s}\right)$ :

$$
u_{i}\left(\boldsymbol{x}_{s}+\boldsymbol{\xi}\right)=u_{i}^{s}\left(\boldsymbol{x}_{s}\right)+\frac{\partial u_{i}^{s}\left(\boldsymbol{x}_{s}\right)}{\partial x} \xi+\frac{\partial u_{i}^{s}\left(\boldsymbol{x}_{s}\right)}{\partial y} \eta+\frac{\partial u_{i}^{s}\left(\boldsymbol{x}_{s}\right)}{\partial z} \zeta+O\left(|\boldsymbol{\xi}|^{2}\right) .
$$

Substituting the surface velocity gradient (20) into equation (28), the surface velocity $u_{i}^{s}\left(\boldsymbol{x}_{s}\right)$ may now be expressed by a following quadratic polynomial 


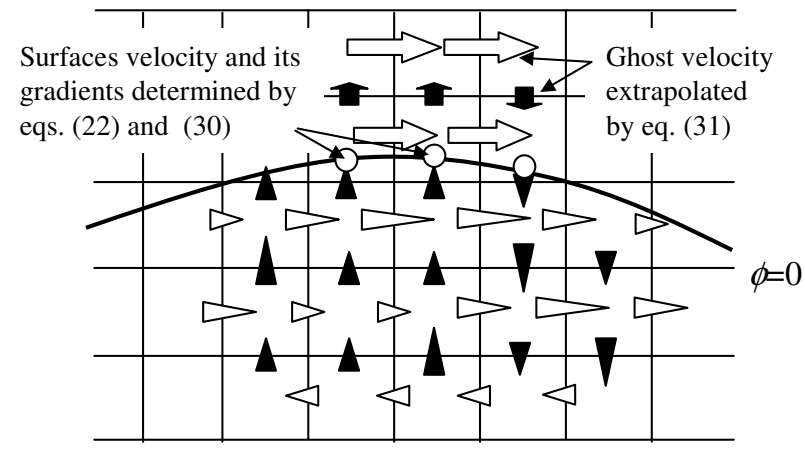

Fig. 2. Extrapolations of velocity and its gradients from grids near the surfaces to the surface location and the empty grids.

$$
\begin{aligned}
u_{i}^{s}\left(\boldsymbol{x}_{s}\right) & =u_{i}\left(\boldsymbol{x}_{s}+\boldsymbol{\xi}\right)+b_{i 1} \xi^{2}+c_{i 2} \eta^{2}+d_{i 3} \zeta^{2}+\left(c_{i 1}+b_{i 2}\right) \xi \eta \\
& +\left(d_{i 1}+b_{i 3}\right) \xi \zeta+\left(d_{i 2}+c_{i 3}\right) \eta \zeta+\left(a_{i 1}-\frac{\partial u_{i}\left(\boldsymbol{x}_{s}+\boldsymbol{\xi}\right)}{\partial x}\right) \xi \\
& +\left(a_{i 2}-\frac{\partial u_{i}\left(\boldsymbol{x}_{s}+\boldsymbol{\xi}\right)}{\partial y}\right) \eta+\left(a_{i 3}-\frac{\partial u_{i}\left(\boldsymbol{x}_{s}+\boldsymbol{\xi}\right)}{\partial z}\right) \zeta
\end{aligned}
$$

where the local coefficients $a_{i j}$ to $d_{i j}$ have determined by solving equation system (25).

In this way, the surface velocity and its gradients can be determined to fulfil the zero tangential shear boundary conditions, approximately, at the surface (see figure 2).

\subsection{Ghost velocity}

In order to compute the advection equations (10) and (16) near the freesurface, the fluid velocity in the empty (or ghost) grid cells outside the surface needs to be determined on the fixed grid system. Since the surface velocity and its gradients have been obtained in $\S 2.3$, the ghost velocity at $\boldsymbol{x}=\boldsymbol{x}_{s}+\boldsymbol{\xi}$ ( $\boldsymbol{\xi}$ is now position vector outside surfaces) may also be determined by the quadratic equation (see figure 2),

$$
\begin{aligned}
u_{i}\left(\boldsymbol{x}_{s}+\boldsymbol{\xi}\right) & =u_{i}^{s}\left(\boldsymbol{x}_{s}\right)-b_{i 1} \xi^{2}-c_{i 2} \eta^{2}-d_{i 3} \zeta^{2}-\left(c_{i 1}+b_{i 2}\right) \xi \eta \\
& -\left(d_{i 1}+b_{i 3}\right) \xi \zeta-\left(d_{i 2}+c_{i 3}\right) \eta \zeta+\left(\frac{\partial u_{i}\left(\boldsymbol{x}_{s}+\boldsymbol{\xi}\right)}{\partial x}-a_{i 1}\right) \xi \\
& +\left(\frac{\partial u_{i}\left(\boldsymbol{x}_{s}+\boldsymbol{\xi}\right)}{\partial y}-a_{i 2}\right) \eta+\left(\frac{\partial u_{i}\left(\boldsymbol{x}_{s}+\boldsymbol{\xi}\right)}{\partial z}-a_{i 3}\right) \zeta
\end{aligned}
$$

It should be noted that the extrapolated ghost velocity is continuous across the surface and is consistent with the surface zero tangential shear boundary con- 


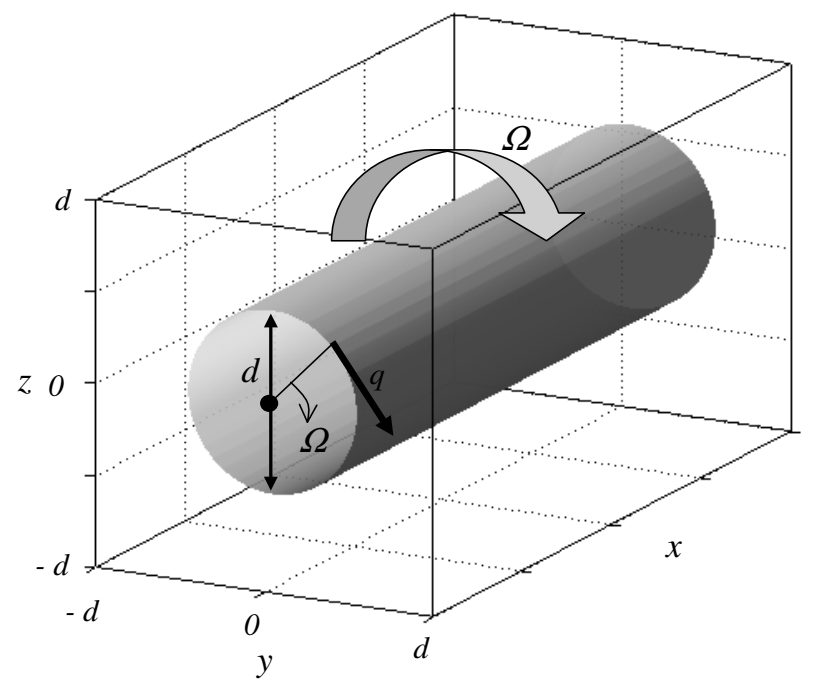

Fig. 3. Computational domain for the numerical test.

dition. Since both equations (30) and (31) can determine spatial variations in sub-surface velocity gradients, they are applicable to a surface boundary layer where high gradients of shear occur, thus presenting an appropriate model for dynamic surface-vortex interactions under droplet impacts.

\section{Validation}

\subsection{Zero tangential shear on the surface}

In order to confirm the present method fulfils the zero tangential shear condition given on the free-surface, the residual error $\epsilon=\left[\left(\partial u_{n} / \partial t+\partial u_{t} / \partial n\right)^{2}+\right.$ $\left.\left(\partial u_{n} / \partial s+\partial u_{s} / \partial n\right)^{2}\right]^{1 / 2}$ on the surface of a rotating fluid cylinder in a Cartesian grid system is considered (see figure 3). If the ghost velocity in the empty grid cells has been properly extrapolated from a fluid containing region where the rotating fluid velocity is specified inertially, it follows that $\epsilon$ at the cylinder surface must be negligibly small. The rotating flow without relative distortion of the fluid element corresponds to a solid-body rotation with angular velocity $\Omega$ and tangential velocity $q$, which ensures the zero tangential stress at the fluid boundary and gives the following surface vorticity $\omega_{s}$ (Longuet-Higgins , 1992).

$$
\omega_{s}=2 \Omega=-2 \kappa q
$$

Fig. 4 (left) shows the maximum and rms of $\epsilon$ on the cylindrical free-surface rotating at $\Omega=2.0$. The computed values of $\epsilon$ are less than truncation precision $\left(10^{-13}\right)$ of the IEEE floating point arithmetic used at any grid size. 

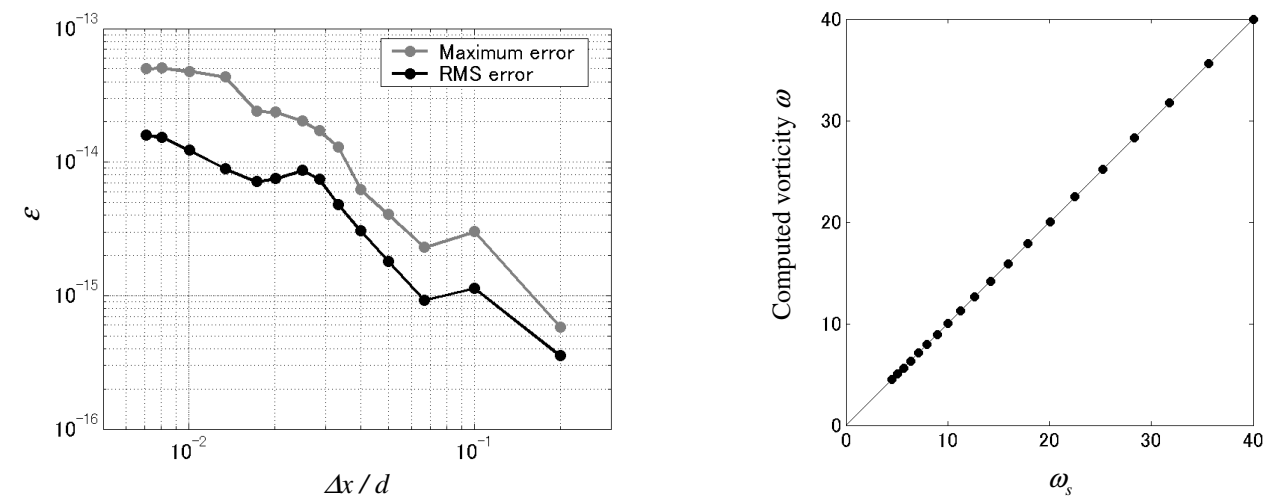

Fig. 4. Residual error of tangential shear on the surface of a water cylinder (left) and a correlation between computed and analytical surface velocities (right).

We thus conclude that the zero tangential shear is achieved by the technique described in $\S 2.3$.

The surface vorticity $\left(\omega=\partial u_{s} / \partial z-\partial w_{s} / \partial x\right)$ can be directly obtained by using the velocity gradients given by equation (20). The computed surface vorticity coincides with the vorticity given by equation (31), demonstrating the proposing technique is capable of estimating appropriate surface vorticity (See Fig. 4 right)

\section{Droplet impacts}

Surface deformations and sub-surface vortex structures under both vertical and oblique impact of droplets has been computed using the present technique. A spherical water droplet of diameter $d^{*}$ with impact velocity $U^{*}$ is splashed at an impact angle $\alpha$ onto a still water pool of water depth $h^{*}$ in the Cartesian staggered grid system (see Fig. 5). Periodic boundary conditions are specified on the side walls, and a non-slip boundary condition is imposed at a bottom of the pool. The water depth is chosen to be sufficiently deep that the effects of the droplet impact are negligible near the bottom. Initial conditions for the droplet impact trials are shown in Table 1.

\subsection{Cavity formation due to vertical droplet impact}

Cavity formation processes under the vertical droplet impacts have been experimentally observed and classified in terms of both the Froude, Fr, and Weber, $W e$, numbers (Liow , 2001). The computed viours of the cavities are compared with the experimental results (see figure 6). The computed results are found to reproduce the splashing process after the impact - initial forma- 

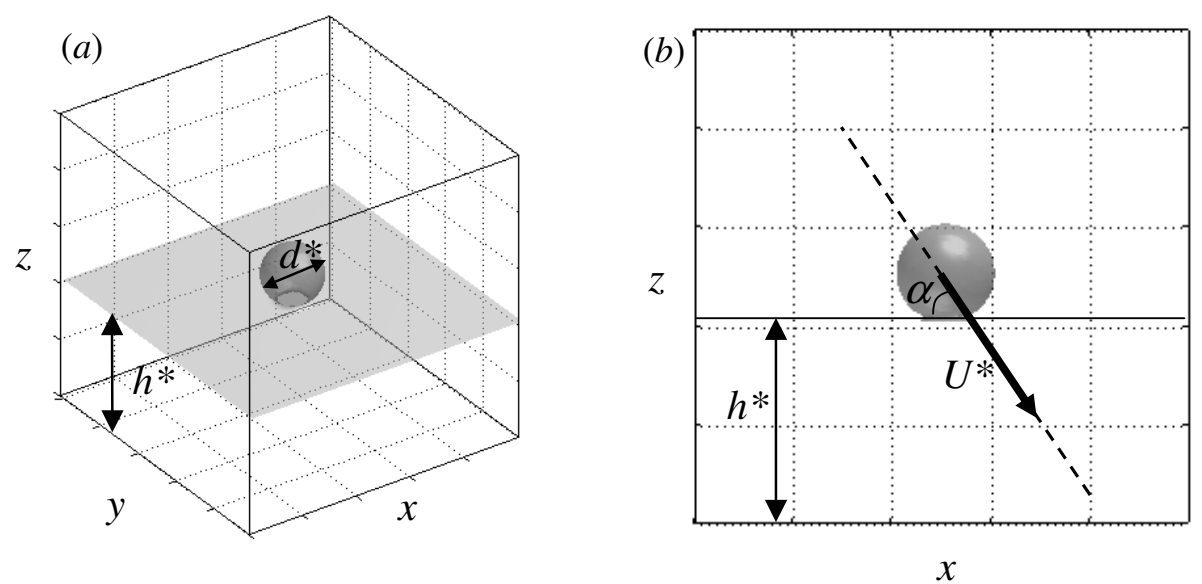

Fig. 5. Computational domain for droplet impacts.

Table 1

Impact conditions

\begin{tabular}{cccccccc} 
Run & $\alpha\left(^{\circ}\right)$ & $d^{*}(\mathrm{~mm})$ & $U^{*}(\mathrm{~m} / \mathrm{s})$ & $W e$ & $F r$ & $R e$ & Grid size $(x, y, z)$ \\
\hline 1 & 90 & 1.86 & 1.29 & 43.5 & 91.3 & 2399 & $40,40,60$ \\
2 & 90 & 2.09 & 1.61 & 75.0 & 126.6 & 3365 & $40,40,140$ \\
3 & 90 & 2.12 & 2.13 & 165.4 & 218.4 & 4516 & $120,120,120$ \\
4 & 90 & 1.88 & 2.88 & 268.0 & 450.2 & 5414 & $90,90,180$ \\
5 & 90 & 2.60 & 0.801 & 23.0 & 25.2 & 2083 & $60,60,90$ \\
6 & 19.9 & 1.95 & 2.20 & 162.3 & 253.3 & 429 & $140,100,40$ \\
7 & 19.2 & 1.80 & 2.22 & 152.5 & 279.4 & 400 & $140,100,40$ \\
8 & 7.4 & 1.90 & 2.18 & 155.2 & 255.2 & 414 & $140,100,40$
\end{tabular}

tions of the cavity, radial spreading of the cavity rim and rapid variation of the cavity shape.

The time evolution of dimensionless cavity depths $\left(D_{c}\right)$ has also been compared with Liow's experiments in figure 7 . The cavity depth rapidly increases in the initial growth phase, and then the cavity slowly grows until the bottom of the cavity begins to rise in the collapse phase. The present results show the typical growth and collapse processes associated with cavities and are identical to the experimental results across the range of Froude and Weber numbers. These results show our computations of the dynamic free-surface responses to the droplet impacts are appropriate. 

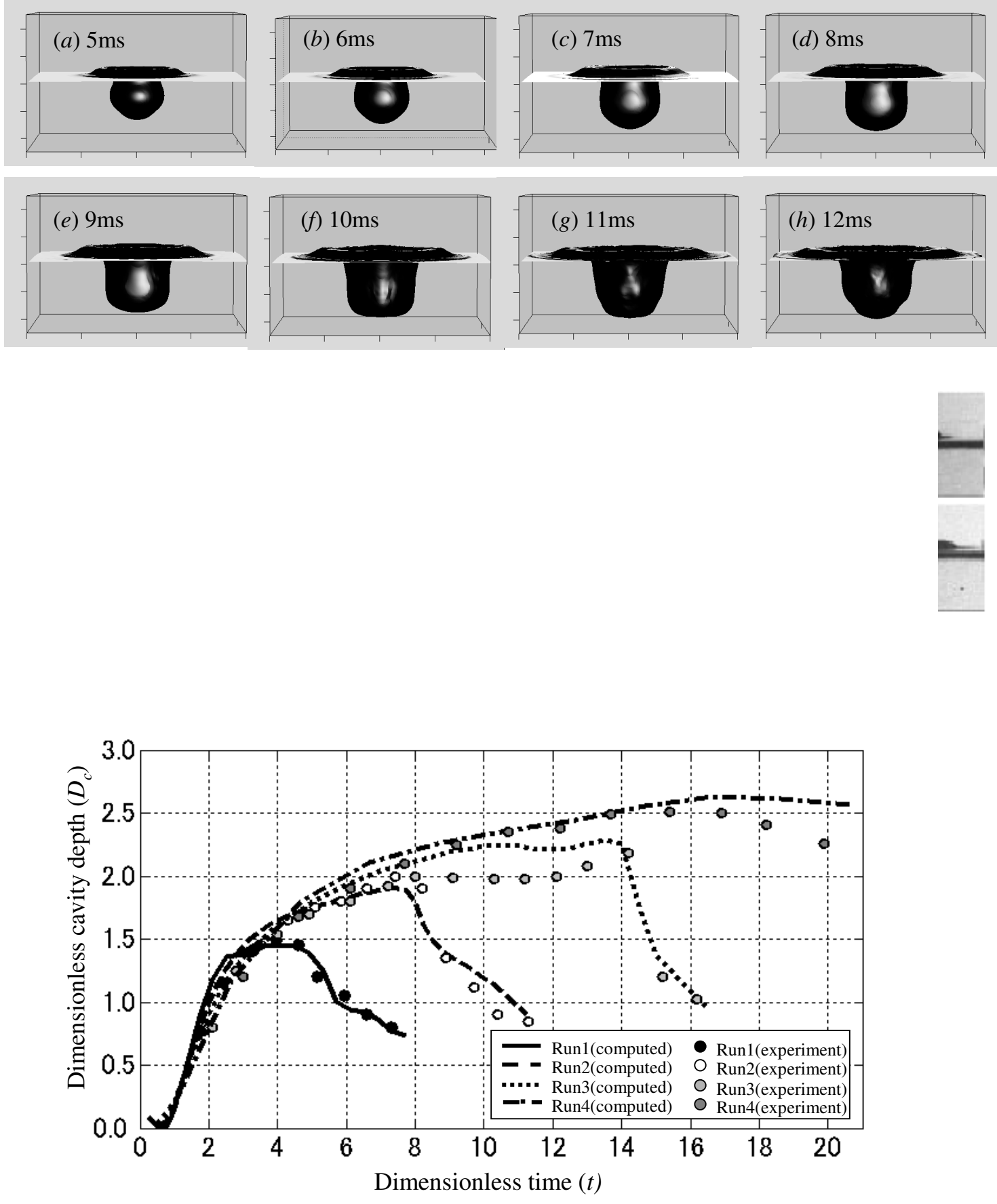

Fig. 7. Computed $(-)$ and observed $(\bullet)$ dimensionless cavity depth versus dimensionless time ; $(a)$ : Run 1; (b): Run 2; $(c)$ : Run 3; $(d)$ : Run 4.

\subsection{Evolution of the vortex ring}

When a droplet touches a still water surface, vorticity is produced at the contact surface between the droplet and receiving surface where there is a high curvature of the free-surface, as described by equation (31). As the droplet 

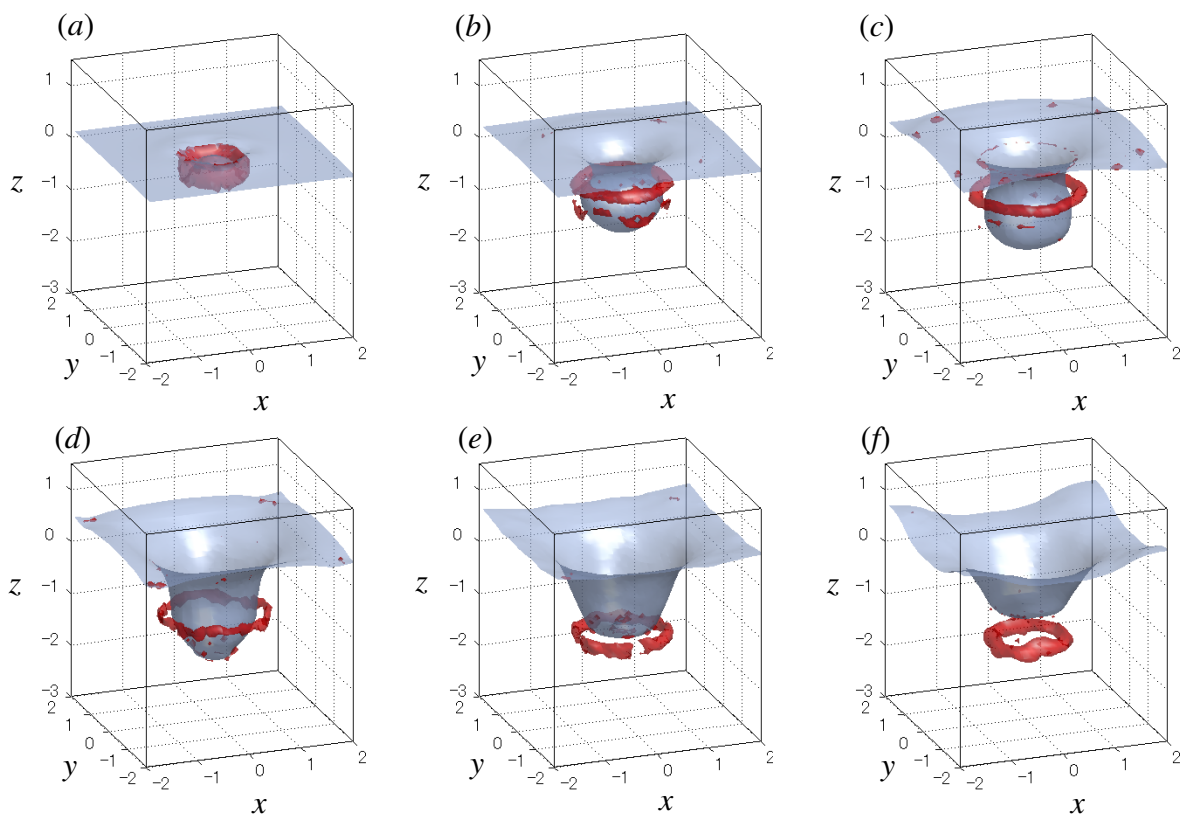

Fig. 8. Evolution of the vortex core representing the primary vortex ring in Run 2. The vortex core is identified on the basis of the, so-called, $\lambda_{2}$ method (Jeong and Hussain , 1995). (a) $2.16 \mathrm{~ms}$ after the impact, (b) $6.49 \mathrm{~ms}$, (c) $10.82 \mathrm{~ms}$, (d) 15.15 ms, (e) $19.48 \mathrm{~ms},(e) 23.80 \mathrm{~ms}$.

penetrates the receiving water surface vorticity is developed along the circumference of the contact plane until the droplet is fully immersed, thus resulting in the formation of an axisymmetric vortex ring beneath the free-surface (see figure $8 a$ ). As the cavity deepens the vortex ring surrounding the cavity wall is displaced downward (see figure $8 b-d$ ). Finally, during the collapse of the cavity, the vortex is separated and ejected from the bottom of the cavity to be displaced downward via self-induction (see figure $8 e-f$ ).

While typical behaviour of the vortex ring may be assumed to be axisymmetric, two-dimensional, motion until the vortex ring separates from the cavity as shown in figure 8 . Three-dimensional deformations of the ring have been also observed at low $\mathrm{Fr}$ and $W e$ in visual observations of a dye droplet by Peck and Sigurdson (1994). According to their study, azimuthal instability develops along the concentric vortex ring after the ring is released from the cavity, and the azimuthal perturbations are periodically amplified on the vortex axes producing counter-rotating vertical vortex pairs wrapped by the primary vortex ring. A complex vortex structure involving 'petals' (hairpin vortex around the ring) and a 'stalk' (a vertical vortex which connects the free-surface and the ring) has been speculated to be formed in their study.

Figure 9 shows sequential distributions of numerically dyed droplet fluid (represented as an iso-surface of dye density of 0.1) and iso-surfaces of the vertical vorticity under the same droplet conditions as the experiments by Peck and Sigurdson (1994). The evolution of the three-dimensional vortex field is found 


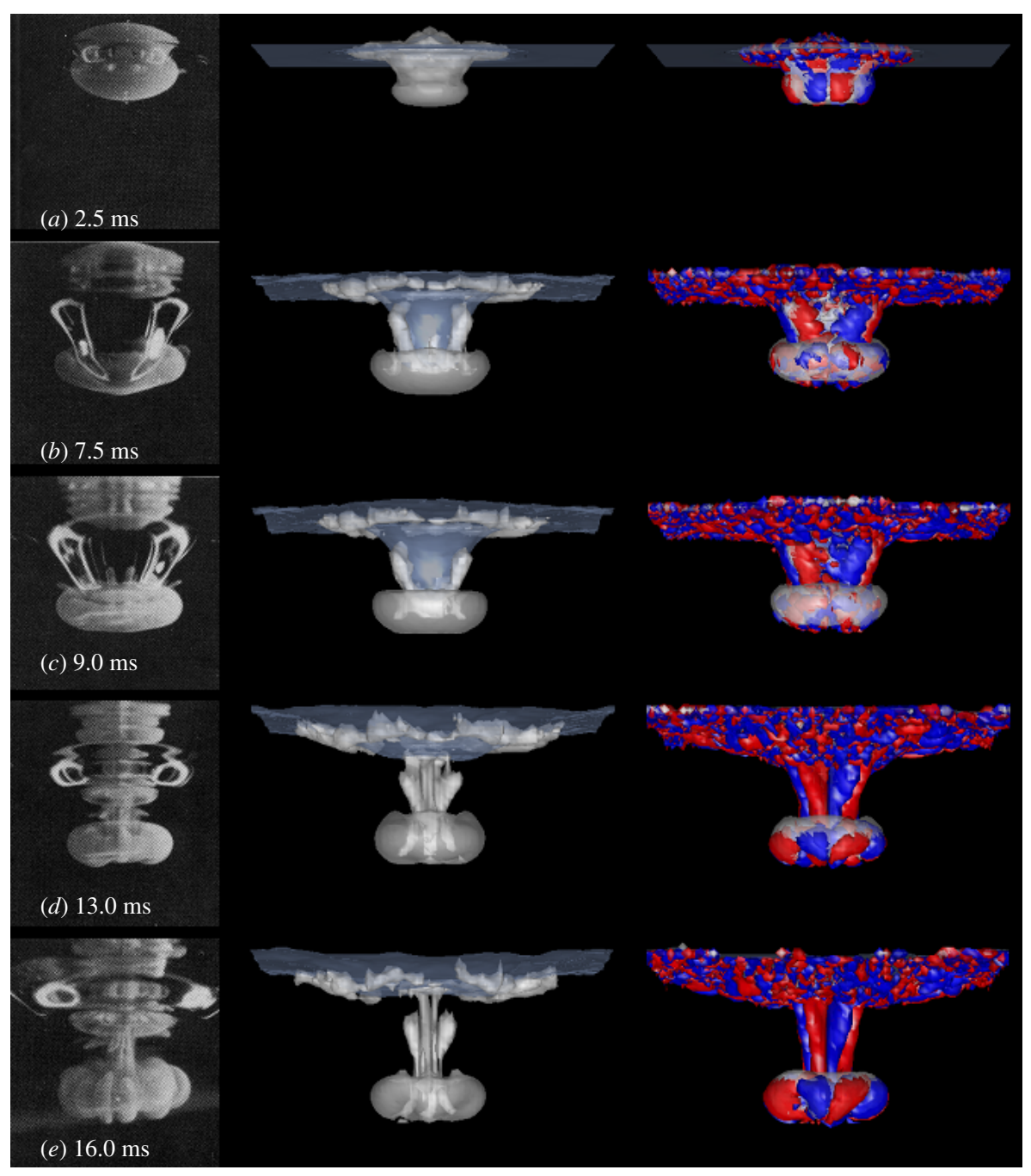

Fig. 9. Experimental images of dyed fluid (left, after Peck and Sigurdson (1994)), distributions of the computed numerical dye (middle) and iso-surfaces of the computed vertical vorticity (right) in the corresponding phases (Run 5); red: +0.1 , blue: -0.1, white: primary vortex ring. The results continues to figure 10 .

from the computed results as follows. While the primary concentric vortex ring is developed on the cavity bottom at an early stage of the droplet impact, four pairs of counter-rotating vertical vorticites are simultaneously formed in the vicinity of the vortex ring (figure $9 a$ ). The vortex pairs are stretched downward as the primary vortex ring is displaced, evolving into a counter-rotating vortex layer enveloping the cavity wall (figure $9 b$ and $c$ ). During the cavity collapse phase, the vortex layer is focused on the central axis of the vortex ring between the descending ring and the reversing cavity surface, and is vertically stretched between the descending ring and the reversing cavity surface along the central axis of the vortex ring. The counter-rotating vortices extending from the cavity are intensified on the axis via a, typical, stretch-and-intensification process (figure $9 d$ and $e$ ). The bottom ends of the counter-rotating vortices are wrapped around the rotating vortex ring and are pulled out from the hole 


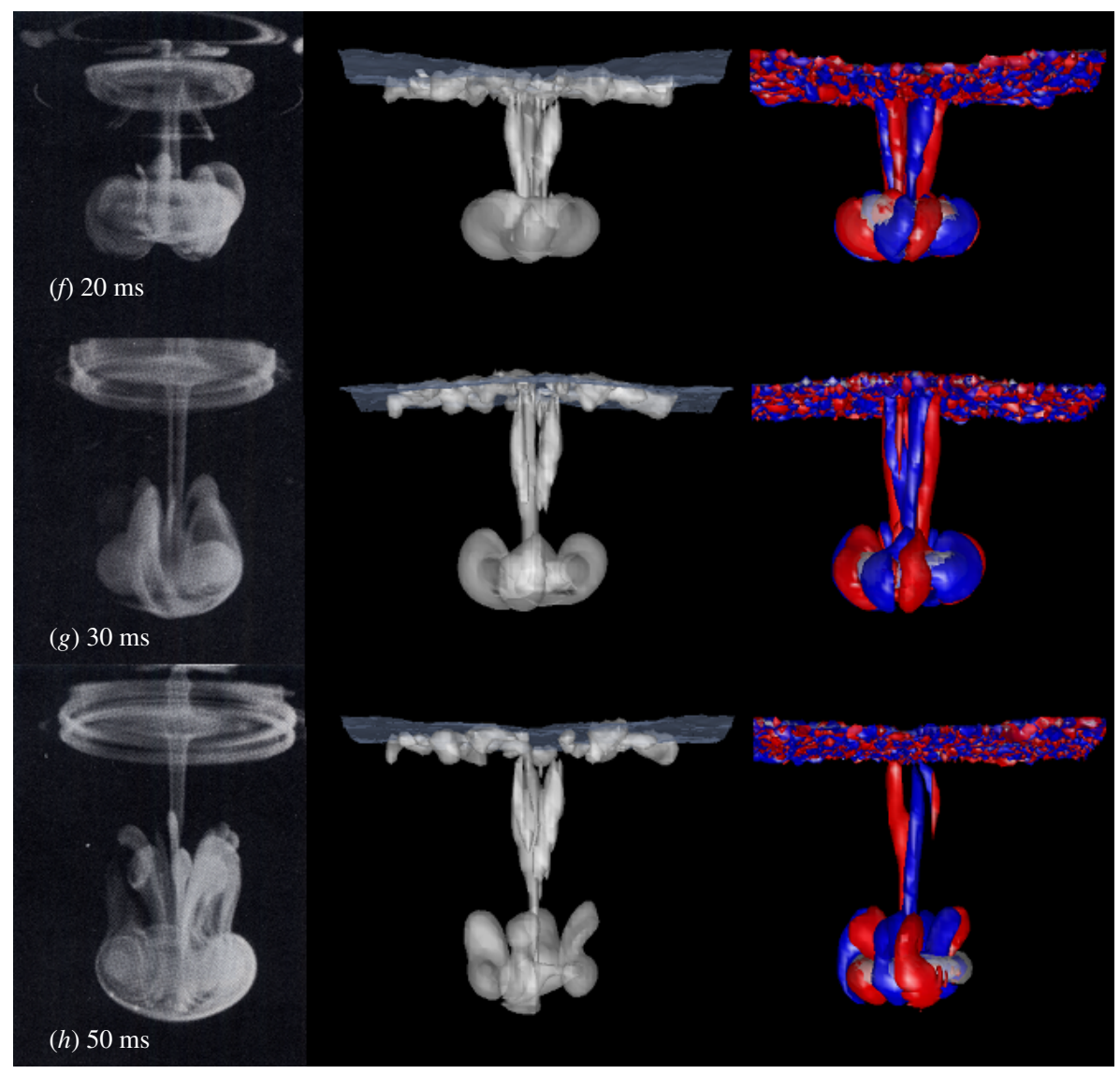

Fig. 10. See a caption of figure 9 .

in the ring (Fig. $9 d-e$ and Fig. $10 f-h$ ). The behaviours of dyed fluid in the experiment by Peck and Sigurdson (1994) are well reproduced by the present results. They clearly show how the vertical counter-rotating vortices form the 'stalk' which has been observed in the experiment. This 'stalk' is formed along the central axis by dye entrained in the vortices which are stretched between the cavity bottom and the vortex ring. Furthermore, the wrapping process of the counter-rotating vortices around the primary vortex ring causes formations of four pairs of 'petals', which are also observed in the numerical dye trapped within the vortices as shown in figure 10 .

Although it is not possible to compare the computed free surface with the experimental images, since the the locations of the surface are not identified in the published experimental photographs, comparisons can be made between the distribution and extent of the experimental and numerical dyes. The distributions of the both numerical and experimetal dyes are highly correlated in the early stage of the formation of the vortex ring (see figure $9 a-d$ ). The azimuthal undulations of the numerical dye appear to form 'petals' which are identical with the experimental dye distribution (see figure $9 e-10 f$ ). While the numerical dye trapped within the counter-rotating vortices is released from the ring (in an analogous manner to the experiment) leading to vertical growth 
(a) $2.5 \mathrm{~ms}$

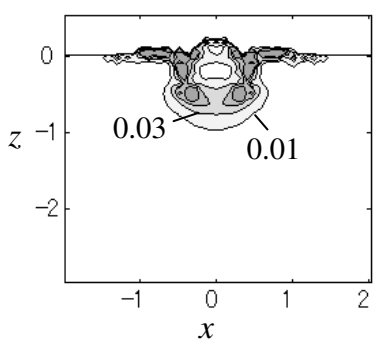

(e) $16.0 \mathrm{~ms}$

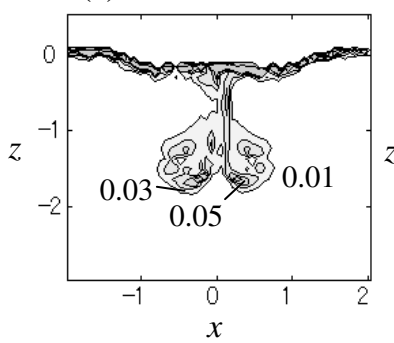

(b) $7.5 \mathrm{~ms}$

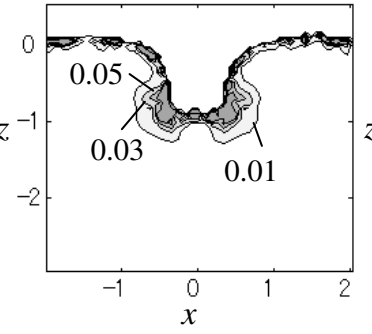

(f) $20 \mathrm{~ms}$

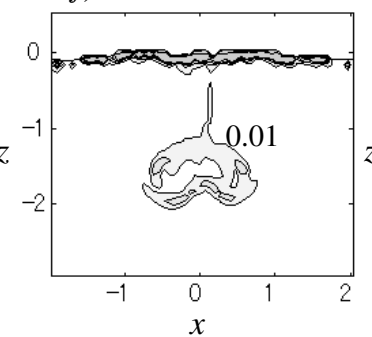

(c) $9.0 \mathrm{~ms}$

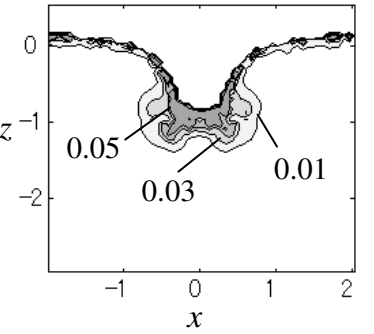

(g) $30 \mathrm{~ms}$

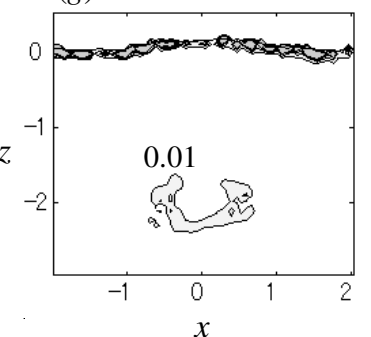

(d) $13.0 \mathrm{~ms}$

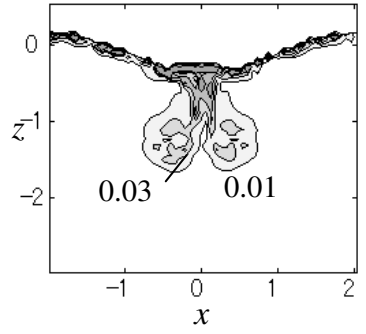

(h) $50 \mathrm{~ms}$

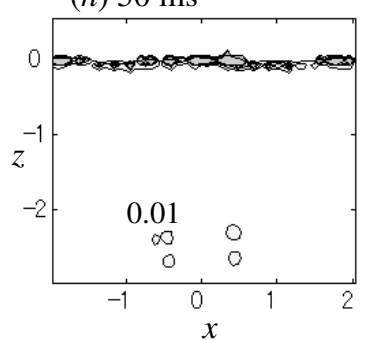

Fig. 11. Evolution of subgrid scale turbulent energy in Run5.

of the 'petals', the computed 'petals' are shorter than the experimental ones in the later stages of development (see $10 g-h$ ). According to Peck and Sigurdson (1994), the number of 'petals' shed was observed to vary from three to five during the experiments, This suggests the mode of amplification of the azimuthal instability is very sensitive to initial conditions, and the subsequent evolution of the three-dimensional vortex may alter during experimental trials. Therefore, a quantitative comparison of the instantaneous flow field in this phase is likely to be difficult. However, the computed results provide a reasonably interpretation of the overall features of the two- to three-dimensional transitions of the vortex structure under droplet impacts.

Figure 11 shows cross-sectional distributions of the subgrid scale (SGS) turbulent energy at the same phases as in figures 9 and 10. The SGS energy produced at the impact is concentrated in the vicinity of the bottom edges of the cavity, and then it is released from the cavity and descends with the vortex ring when the cavity collapses (see figure $11 b-d$ ). The SGS energy is intensified between the ring and the reversing cavity (see figure $11 c$ and $d$ ), while the turbulence within the ejected ring is monotonically diffused (see figure 11 $e-f)$.

Figure 12 shows the evolution of the vortex cores and the principal values in the principal axes of strain tensors, representing the directions and degree of stretching that a fluid element receives. In cases where significant threedimensional deformation of the primary vortex ring occurs, i.e. at low Weber and Froude numbers, a shear layer forms where the fluid is highly stretched obliquely in a downward direction, enveloping the cavity wall (figure $12 b$ at $4.325 \mathrm{~ms}$ after the droplet impact), and azimuthal variations of the strain begin to emerge in the region where the vortex ring is located (figure $12 c$ at 

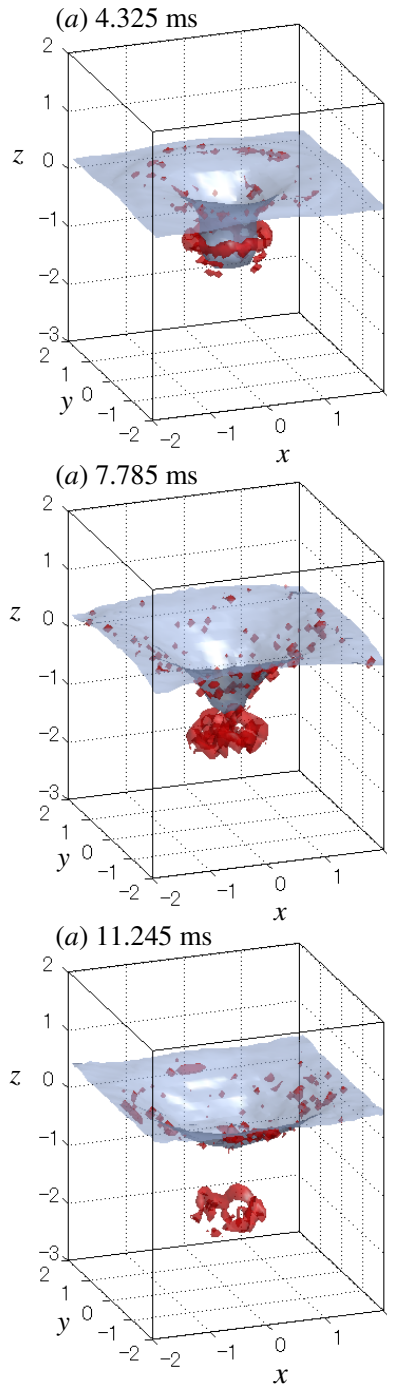
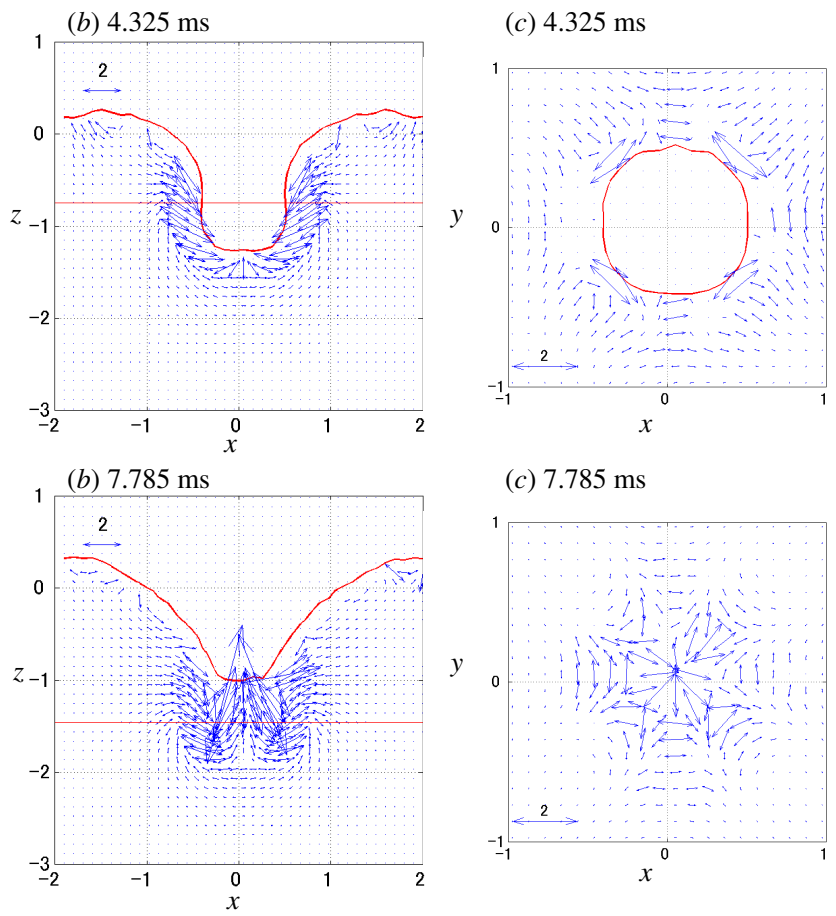

(c) $7.785 \mathrm{~ms}$

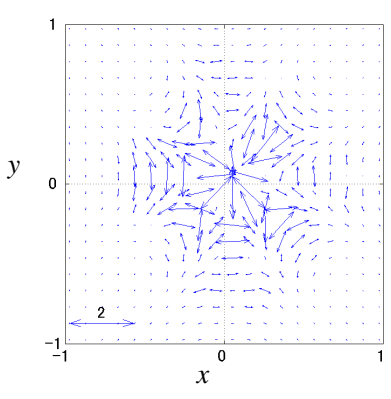

(c) $11.245 \mathrm{~ms}$
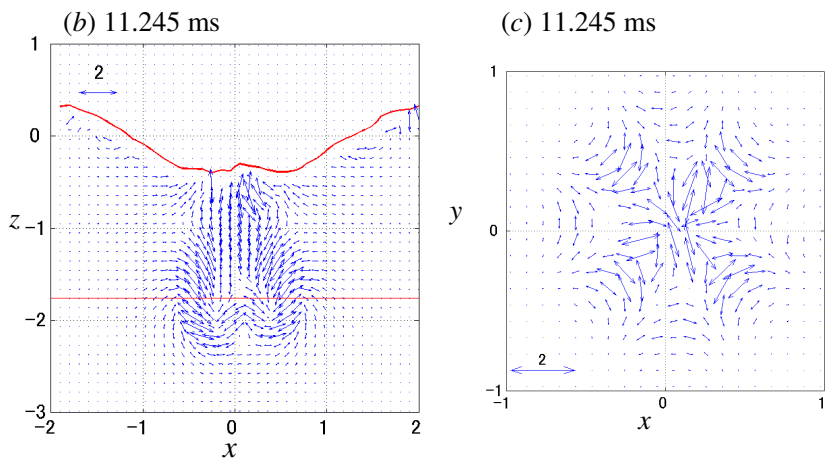

Fig. 12. (a) evolution of vortex cores, $(b)$ principal axes of the strain tensor on a vertical cross-section and $(c)$ principal axes of the strain tensor on a horizontal cross-section at the level of the vortex ring in Run 1.

$4.325 \mathrm{~ms}$ ). Under these conditions, the primary vortex ring is found to become unstable in both its axis of rotation and in the shear layer, suggesting strong stretching around the cavity induce instabilities of the primary concentric vorticity and change orientation of the primary vorticity into the direction of the stretch. This re-orientation process causes formation of counter-rotating vorticities enveloping the cavity wall, as previously explained (see also figures 9 and 13a). As the cavity grows quickly downwards a vertical shear layer covering the cavity wall forms. There is a sufficiently high shear gradient in the surface layer, where the free-surface is subjected to the zero tangential shear condition, for strong stretching to occur around the wall (see figure 12 (b) $4.325 \mathrm{~ms}$ ), re-orientating and stretching the vortex filaments and intensified the vortex loops within the surface layer. The vortex ring wraps the bottom bends of the vortex loops resulting from the azimuthal instabilities in the 

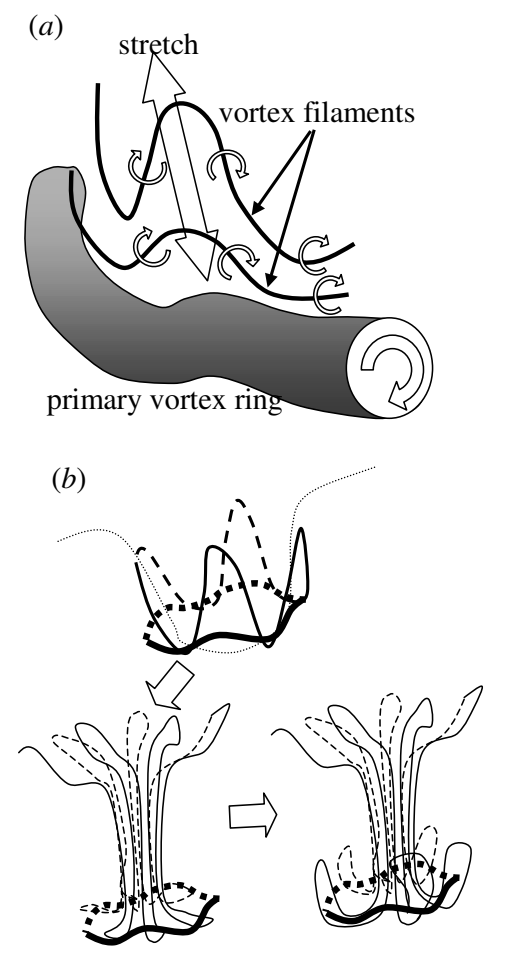

(c)
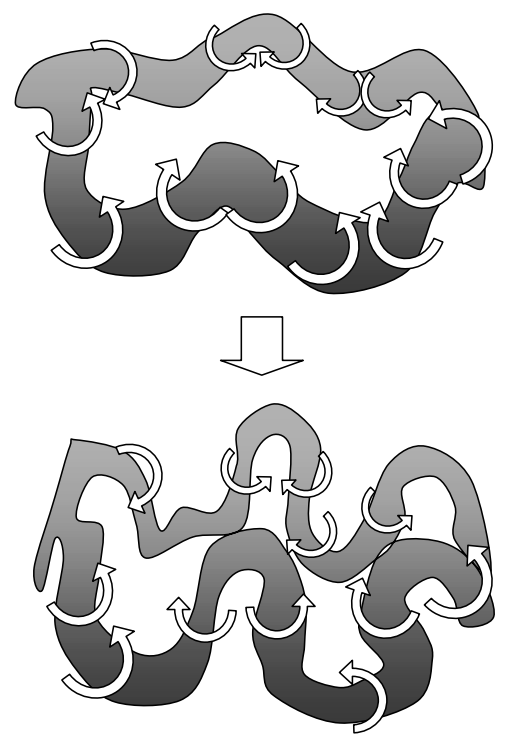

Fig. 13. Schematic representation of $(a)$ the amplification of azimuthal disturbances, (b) a vortex loop stretched in high strain field of the surface layer and a formation of 'stalk' and $(c)$ the three-dimensional deformation of the primary vortex ring.

surface layer and pulls them downward. While the upper bends adhering to the surface layer are pulled upward as the cavity bottom reverses (see figure 13b). The wrapped bottom bends of the vortex loop are rolled up by the primary vortex ring and are left behind by the descending ring. As the cavity collapses (7.785 ms after the impact), vertical stretching between the primary ring and reversing cavity bottom become significant resulting in amplification of the undulations in the primary vortex ring (see also figure 13c). In this way, the transition from a single concentric ring vortex to the complex, threedimensional, wavy vortex structure, triggered by the azimuthal instability, occurs within the sub-surface shear layer.

\subsection{Oblique droplet impact}

Patterns of local surface deformation and spreading of droplet fluid under oblique droplet impacts at various impact angles have been studied experimentally by Leneweit et al. (2005). In this section the reproducibility of our computation under the same impact conditions is discussed together with new findings on the three-dimensional vortex structures beneath the impact cavities. 

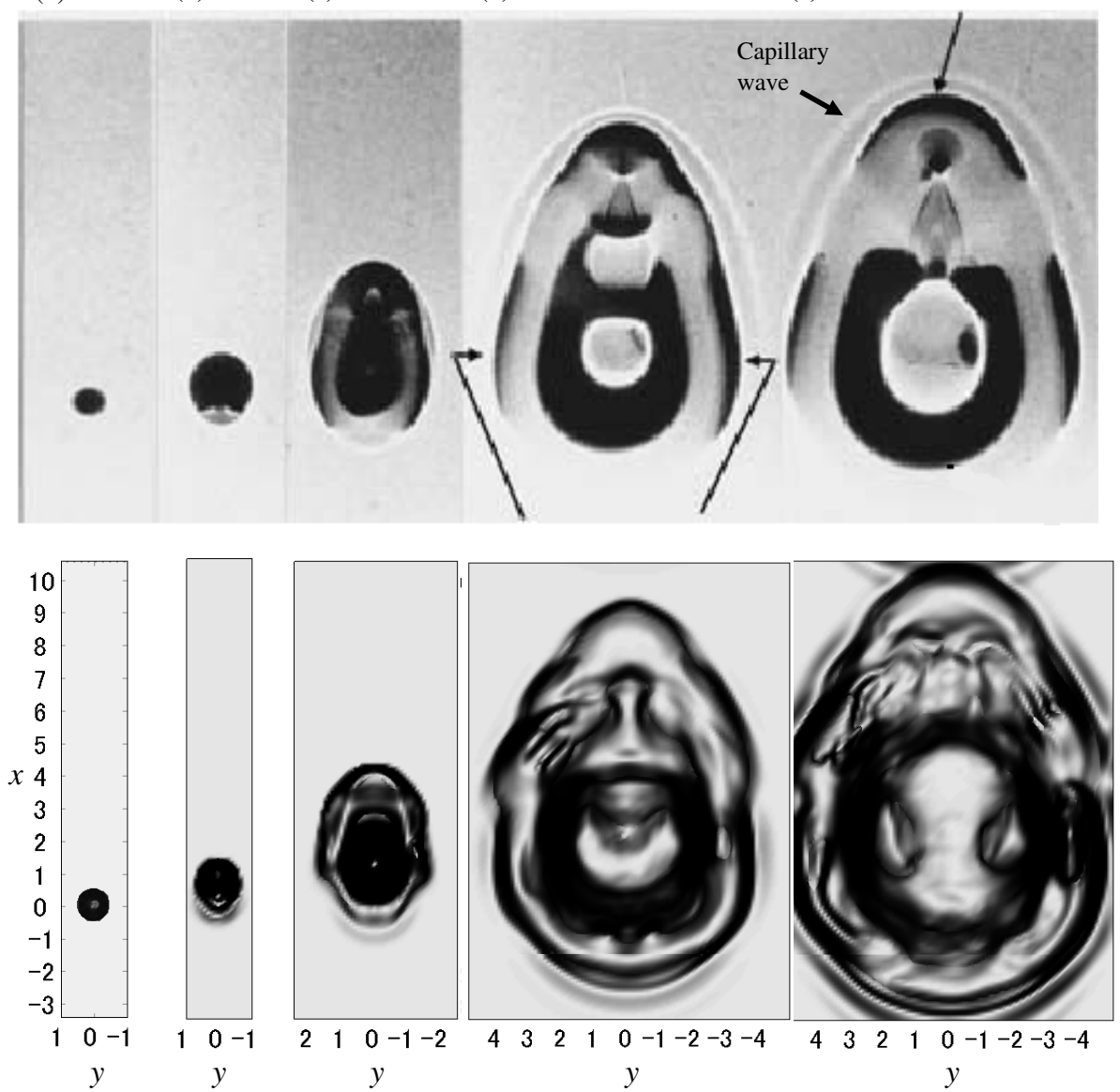

Fig. 14. Oblique droplet impacts from Run 6, seen from a vertical perspective; top: experimental shadow-graph images (Leneweit et al. , 2005), bottom: computed surface deformation; (a) dimensionless time after impact $t=-0.2,(b) 0.8,(c) 4.0,(d)$ 15.7, (e) 21.0.

The sequence of shadow-graphs of the cavity from a vertical perspective taken by Leneweit et al. (2005) are compared with visualised computational results in figure 14. To aid comparison the computational images have been scaled to be equal to experimental photographs. In the experimental results the surface deformation is visualised using a shadow-graph of refracted light at the free surface, while the computed free surface is represented by the reflection of an "overhead" lighting at the surface using a standard computer graphics lighting model. In both case, however, dark areas indicated deformed or sloping parts of the free surface, where the reflected light is scattered away from the viewer, while bright areas indicate a planar horizontal region of the free surface. Major features observed in the deformation patterns, including the shape of the outer rim of the cavity and the series of capillary waves spreading around the cavity, are found to be identical (see figures 14 and 16).

The growth of the cavity, formation of lamella in the front edge and the spreading wave front from a lateral perspective are also in good agreement with the 


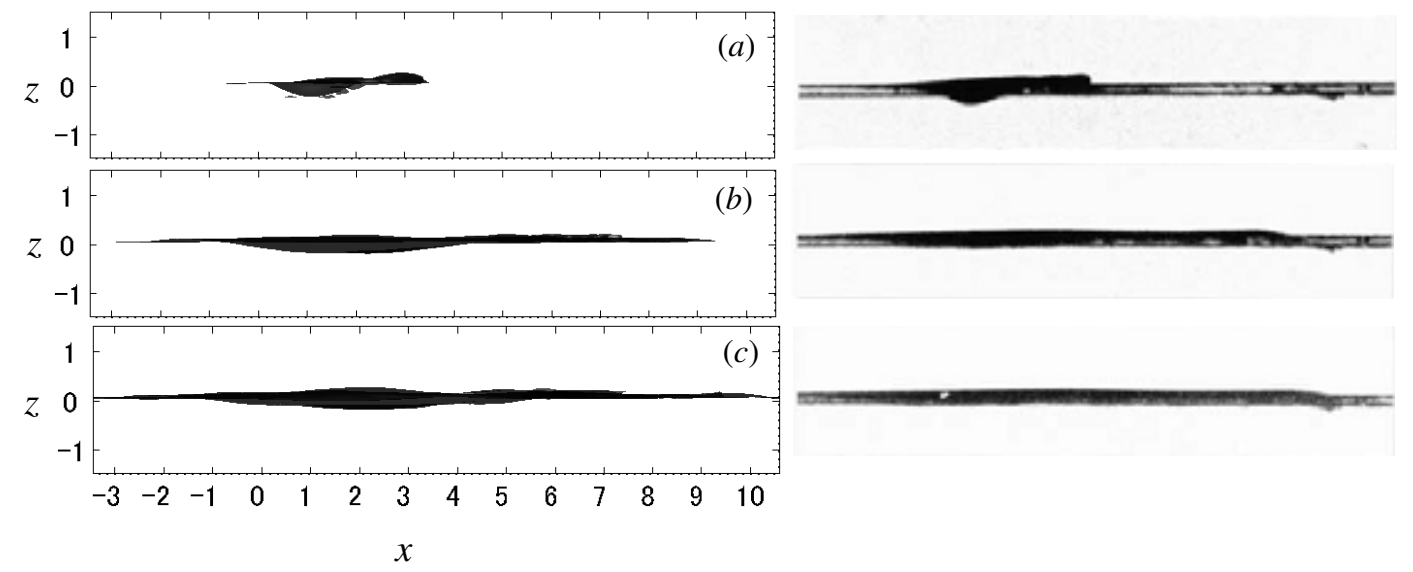

Fig. 15. Lateral view of the oblique droplet impact in Run 7; left: computed surface deformation, right: experimental shadow-graph images (Leneweit et al. , 2005); (a) dimensionless time after impact $t=3.2,(b)$ 15.5, (c) 20.7.
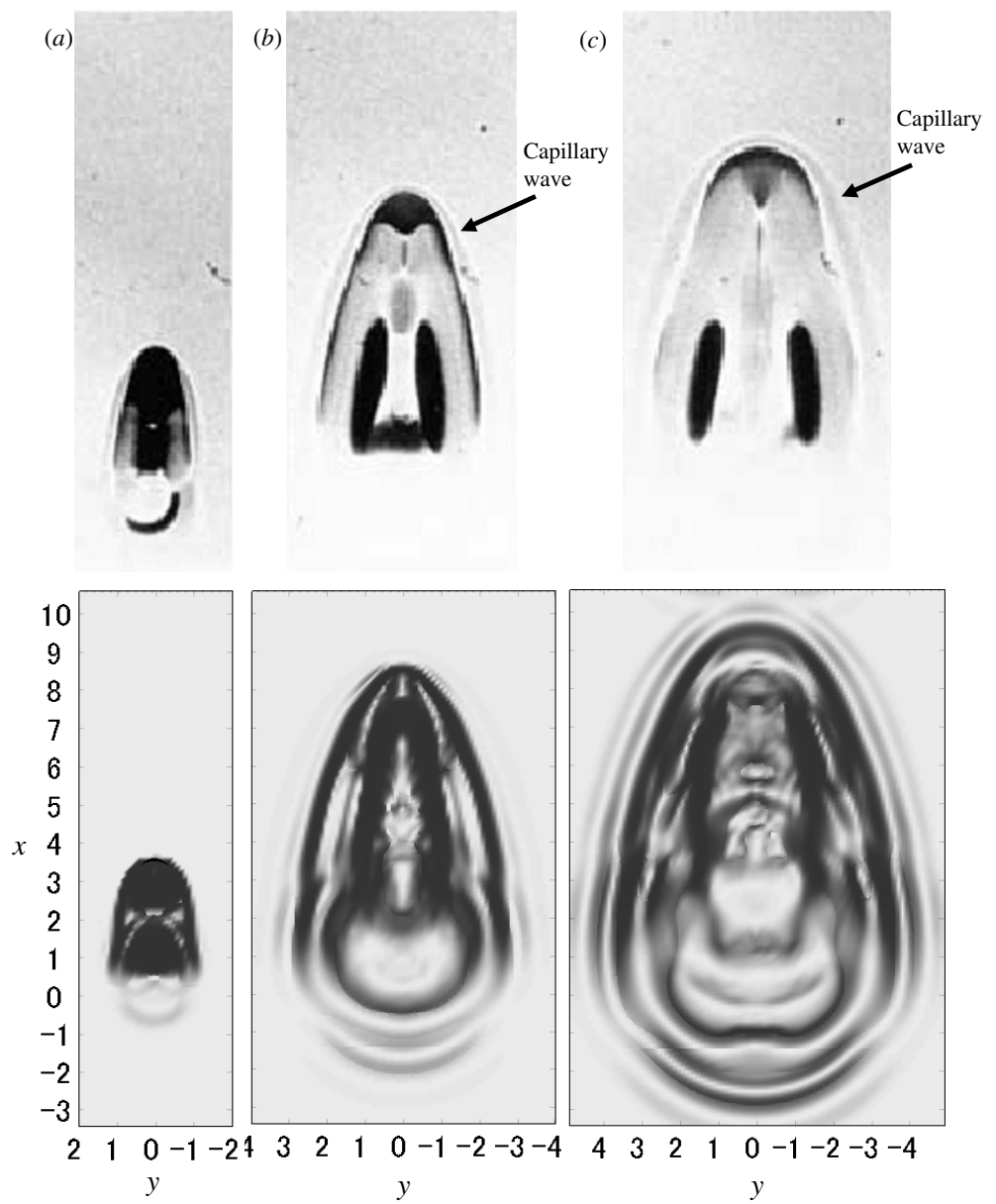

Fig. 16. Vertical perspective of the oblique droplet impact in Run 8; top: experimental shadow-graph images (Leneweit et al. , 2005), bottom: computed surface deformation; $(a)$ dimensionless time after impact $t=3.2,(b) 11.9,(c)$ 17.3. 

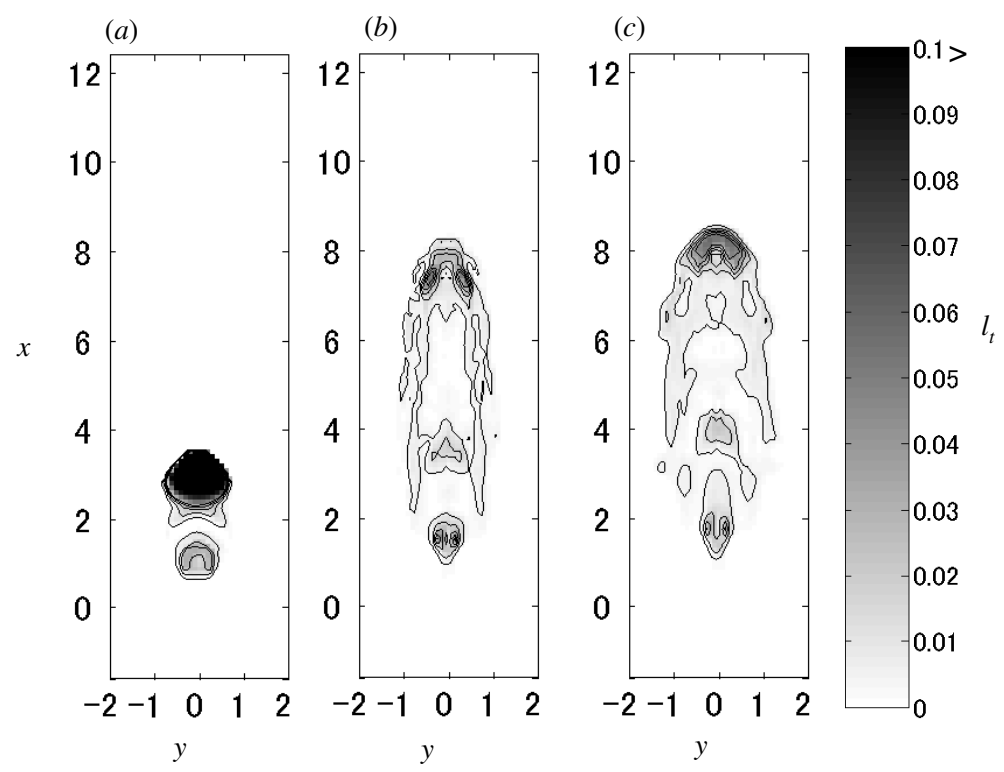

Fig. 17. Thickness of the layer of computed droplet fluid in Run 8; dimensionless time after impact $t=(a) 3.2,(b) 11.9,(c)$ 17.3. Contour interval: 0.005.

experimental results (Figure 15), which leads us to conclude the computations give reasonable reproducibility of the impact process. At small impact angles, $\alpha$, the cavity becomes more prolate and capillary waves spread normal to the cavity rim (see figure 16), these findings are also in agreement with the interpretation presented in Leneweit et al. (2005). Leneweit et al. (2005) also found the inherent spreading patterns of the droplet fluid appear within a thin layer on the surface once it has come to rest after impact. This pattern is formed through fluid convection around the cavity, and therefore is sensitive to local fluid motion near the free-surface. In the present computation the concentration of a passive numerical dye, whose initial concentration $c=1.0$ in the droplet, was used to trace the droplet fluid. Since Leneweit et al. (2005) state that the darkness of the dye in the experimental image is proportional to thickness of the dye layer, the corresponding layer thickness $\left(l_{t}\right)$ has been estimated in the computation using the relation

$$
l_{t} / d=\int c d z
$$

Following the impact the droplet partially coalesce with receiving water to form thin dye layer adjacent to the surface. This results in the formation of longitudinal spreading patterns, which are typically observed from a vertical perspective (see figure 17). In the experimental images the dye cannot be rigorously distinguished from shadows of cast by the deformed surface unless the surface is either located sufficiently distant from the dye or the surface perturbations have disappeared. Consequently, quantitative comparisons between the experimental dye spread and the simulations cannot be made precisely along the center line of the cavity. However, the overall agreement between 


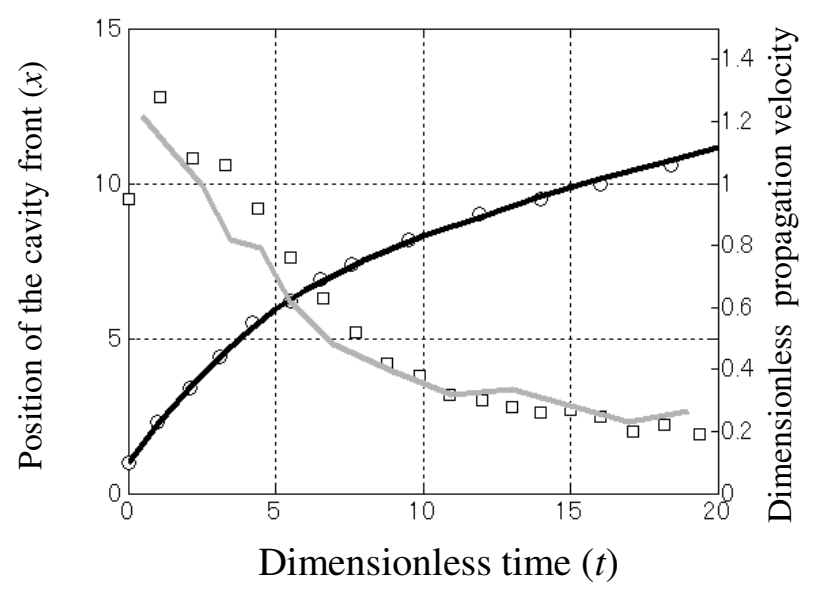

Fig. 18. Comparisons of observed and computed position and velocity of the wave front.

the observed features, such as front shape of the dyed fluid and longitudinal patterns extending from the plunging point to the wave front, suggests the local fluid motion in the vicinity of the free-surface has been computed correctly.

A quantitative comparison of the locations of the propagating cavity front with the experiment by Leneweit et al. (2005) is shown in figure 18. Both the computed front position and front velocity during propagation coincide with the experiment results. The close correlation in the series of comparisons between the experimental of oblique impact and the associated computational results clearly demonstrates the applicability of the present numerical method to the study of free-surface impacting flows.

The sub-surface vortical structures generated by the droplet impact determine both the local convection of free-surface and the resulting patterns of the droplet fluid dispersal. As with the vertical impacts, the initial generation of the vortex ring occurs circumferentially on the contact plane between the droplet and receiving fluid surface, where the surface curvature is high. The initial ring vortex is subsequently released from the droplet and progresses over the undisturbed water surface (see figure $19 a$ and $b$ ). The droplet fluid continues propagating, into the water, with partial immersion and consequently produces a new ring vortex below the surface, since the contact between the quiescent fluid and the bottom of the droplet is still highly curved. The vortex production process continues until the droplet is fully immersed and a borelike wave front is observed (figure $19 a$ to $g$ ). The front and rear parts of this new vortex ring are stretched forward and backward, respectively, between the propagating droplet and previous vortex ring, causing the new ring vortex to deform elliptically. The induced velocity caused by the interaction between the first and second ring vorticies causes the rear part of the vortex ring to be displaced upwards towards the surface, where it is entrained. This leads to the development of a highly deformed surface feature (the scar), see figures 14 

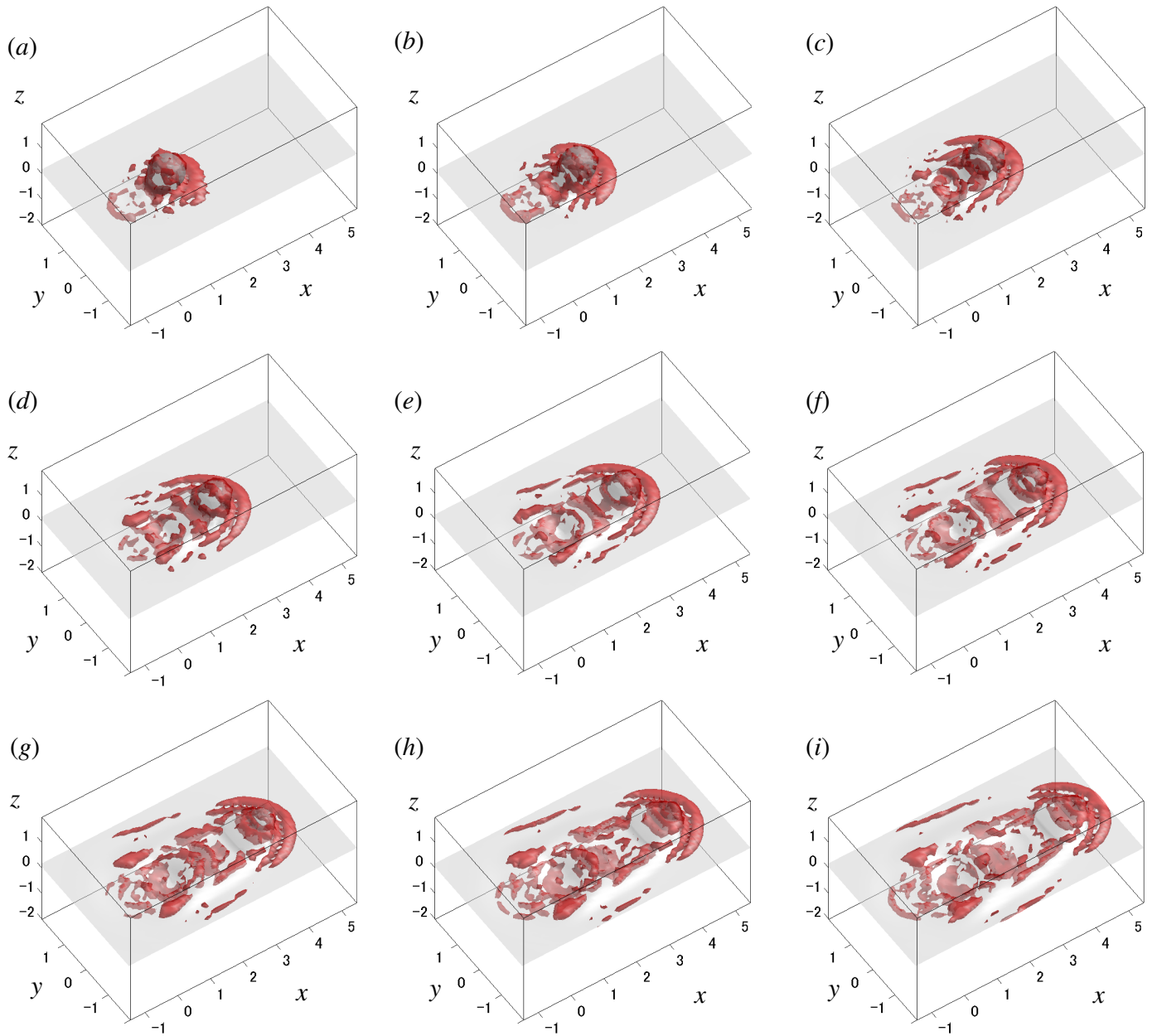

Fig. 19. Evolution of the complex, three-dimensional structures of vortex cores in Run 8; phase interval: $t=0.5$. The vortex core is identified on the basis of the $\lambda_{2}$ method (Jeong and Hussain, 1995).

and 16. While the vortex production is due to curved surfaces of the droplet and receiving surface, the resulting vortices affect local surface deformations to form the scars, indicating the surface-vortex interaction has an important role in determining the impacting process.

\section{Conclusions}

A numerical technique which fulfils the free-surface boundary conditions in a fixed Cartesian grid system has been presented. This technique has been incorporated into a level-set method and applied to the study of turbulent, free-surface, flows under droplet impacts. The resulting fluid motions are governed by both surface/vortex and vortex/vortex interactions as well as by the gravity-dominated cavity formation processes. The computational method has 
been validated by a series of comparisons with visual observations from experiments of cavity formation and the associated vortical structures under both vertical and oblique impacts.

A mechanism which leads to the formation of a fully three-dimensional vortex structure after the vertical impact of a droplet has been identified. In this process, Firstly, the concentric primary vortex filaments become highly stretched around the cavity wall, and then they become unstable through the perturbation of the axis of rotation. These perturbations are amplified concentrically downward on in the shear layer covering the inner cavity, resulting in the formation of counter-rotating vertical vortices. During the cavity collapsing phase, strong stretching between the reversing base of the cavity and the descending vortex ring intensifies the counter-rotating vortex pairs, whose upper ends are bounded by the cavity base and lower ends are rolled up around the primary vortex ring. The transitions to this three-dimensional vortex structures are consistent with 'stalk' and 'petals' patterns of identified in dyed fluid by Peck and Sigurdson (1994).

Under oblique impacts, an inherently three-dimensional vortex structure is formed which depends on the specific impact angle. At small impact angles, a series of vortex rings are produced consecutively at the highly curved contacts between the droplet and the receiving surface until the droplet is fully coalesced. The vortex rings produced by this process are left behind the propagating droplet front and are, subsequently, displaced by the induced velocity caused by the interaction with the previous ring. This process results in a sub-surface symmetric vortex structure which extends in the direction of wave propagation. At large impact angles only a single ring vortex is formed because the droplet fluid rapidly coalesced with the receiving water and is entrained into the vortex ring.

\section{Acknowledgements}

Financial support for this study was provided by JSPS Grants-in-Aid Scientific Research (18760368), and a part of this research was done in JSPS Japan UK bilateral research program.

Dr Ingram wishes to acknowledge support for his position from the Scottish Funding Council and for their support of the Joint Research Institute in Energy with the Heriot-Watt University as a component part of the Edinburgh Research Partnership. 


\section{References}

Chang YC, Hou TY, Merriman B, and Osher S 1996, A Level Set Formulation of Eulerian Interface Capturing Methods for Incompressible Fluid Flows, J.Comput.Phys. 124, 449-464.

A computer study of finite amplitude water waves, J. Comp. Phys., 6, 68-94.

Christensen E D and Deigaard R, , Large eddy simulation of breaking waves, Coastal Eng. 42, 53-86.

Esmaeeli A and Tryggvason G 1998, Direct numerical simulations of bubbly flows. Part1. Low Reynolds number arrays, J. Fluid Mech. 377, 313-345 (1998).

Esmaeeli A and Tryggvason G 1999, Direct numerical simulations of bubbly flows. Part2. Moderate Reynolds number arrays, J. Fluid Mech. 385, 325358.

Fedkiw R, Aslam T, Merriman B and Oshear S 1999, A non-oscillatory Eulerian approach to interfaces in multimaterial flows (the ghost fluid method), J. Comp. Phys. 152, 457-492.

Gueyffier D, Li J, Nadim A, Scardovelli R and Zalenski S 1999, Volume-of-fluid interface tracking with smoothed surface methods for three-dimensional flows, J. Comp. Phys. 152, 423-456.

Hirt, CW and Nichols BD (1981), Volume of fluid (VOF) method for the dynamics of free boundaries, J. Comp. Phys., 39, 201 - 225.

Jeong J and Hussain, F (1995), On the identification of a vortex, J. Fluid Mech., 285, 69 - 94.

Jeong, J.-T. and H. K. Moffat (1992), Free-surface cusps associated with flow at low Reynolds number, J. Fluid Mech., 241, 1-22.

Josserand C and Zalenski S 2003, Droplet splashing on a thin liquid film, Physics of Fluids 15, 1650-1657.

Leneweit G, Koehler R, Roesner K G and Schafer G 2005, Regimes of drop morphology in oblique impact on deep fluids, J. Fluid Mech. 543, 303-331.

Labourasse E, Lacanette D, Toutant A, Lubin P, Vincent S, Lebaigue O, Cltagirone J-P and Sagaut P 2007, Towards large eddy simulation of isothermal two-phase flows: Govering equations and a priori tests, Int. J. Multiphase Flow 33, 1-39.

Liow J L 2001, Splash formation by spherical drops, J. Fluid Mech. 427, 73105.

Lubin P, Vincent S, Abadie S and Caltagirone J-P 2006, Three-dimensional large eddy simulation of air entrainment under plunging breaking waves, Coastal Eng. 53, 631-655.

Longuet-Higgins M S 1992, Capillary rollers and bores, J. Fluid Mech. 240, 659-679.

Losasso F, Fedkiw T and Osher S 2005, Spatially adaptive techniques for level set methods and incompressible flow, Computers \& Fluids 35, 995-1010.

Morton D, Rudman M and Liow J-L 2000, An investigation of the flow regimes resulting from splashing drops, Phys. Fluids 12, 747-763. 
Osher S and Sethian 1988, Fronts propagating with curvature dependent speed: Algorithms based on Hamilton-Jacobi formulations, J.Comput.Phys. 79, 12.

Peck B and Sigurdson L 1994, The three-dimensional vortex structure of an impacting water drop, Phys. Fluids 6 (2), 564-576.

Popinet S and Zaleski S (2002), Bubble collapse near a solid boundary: a numerical study of the influence of viscosity, J. Fluid Mech., 464, 137 - 163.

Reboux S, Sagaut P and Lakehal D (2006), Large-eddy simulation of sheared interfacial flow, Phys. Fluids 18, 105105.

Scardovelli R and Zalenski S 1999, Direct numerical simulation of free-surface and inter-facial flow, Annu. Rev. Fluid Mech. 31, 567-603.

Sousa FS, Mangiavacchi N, Nonato LG, Castelo A, Tome MF, Ferreira VG, Cuminato JA and McKee S 2004, A front-tracking/front-capturing method for the simulation of 3D multi-fluid flows with free surfaces, J. Comp. Phys. 198, 469-499.

Susman M, Smereka P and Osher S 1994, A level-set approach for computing solutions to incompressible two-phase flow, J.Comput.Phys. 114, 146-159.

Susman M and Fatemi E 1999, An efficient, interface-preserving level set redistancing algorithm and its application to inter-facial incompressible fluid flow, SIAM J. Sci. Comput. 20 (4), 1165-1191.

Yakhot V and Orszag SA (1986), Renormalization group analysis of turbulence (I. Basic Theory), J. Sci. Comp., 1, 3 - 51.

Yarin AL 2006, Drop impact dynamics: splashing, spreading, receding, bouncing..., Annu. Rev. Fluid Mech. (38), 159-192.

Watanabe, Y and Saeki, H (1999), Three-dimensional large eddy simulation of breaking waves, Coastal Engineering Journal, 41, 281 - 301.

Watanabe, Y and Saeki, H (2002), Velocity field after wave breaking, Int. J. Numer. Meth. Fluids, 39, 607-637.

Watanabe Y, Saeki H and Hosking RJ (2005), Three-dimensional vortex structures breaking waves, J. Fluid Mech., 545, 291 - 328. 